This item was submitted to Loughborough's Research Repository by the author.

Items in Figshare are protected by copyright, with all rights reserved, unless otherwise indicated.

\title{
Dissipativity-based consensus tracking control of nonlinear multiagent systems with generally uncertain Markovian switching topologies and event- triggered strategy
}

\section{PLEASE CITE THE PUBLISHED VERSION}

https://doi.org/10.1109/TCYB.2022.3141599

\section{PUBLISHER}

Institute of Electrical and Electronics Engineers

\section{VERSION}

AM (Accepted Manuscript)

\section{PUBLISHER STATEMENT}

Personal use of this material is permitted. Permission from IEEE must be obtained for all other uses, in any current or future media, including reprinting/republishing this material for advertising or promotional purposes, creating new collective works, for resale or redistribution to servers or lists, or reuse of any copyrighted component of this work in other works.

\section{LICENCE}

All Rights Reserved

\section{REPOSITORY RECORD}

Wang, Junyi, Huaguang Zhang, Jun Fu, Hongjing Liang, and Qinggang Meng. 2022. "Dissipativity-based Consensus Tracking Control of Nonlinear Multiagent Systems with Generally Uncertain Markovian Switching Topologies and Event-triggered Strategy". Loughborough University. https://hdl.handle.net/2134/18907721.v1. 


\title{
Dissipativity-Based Consensus Tracking Control of Nonlinear Multiagent Systems with Generally Uncertain Markovian Switching Topologies and Event-Triggered Strategy
}

\author{
Junyi Wang, Member, IEEE, Huaguang Zhang, Fellow, IEEE, Jun Fu, Senior Member, IEEE, Hongjing Liang, \\ Member, IEEE, Qinggang Meng, Senior Member, IEEE
}

\begin{abstract}
This article focuses on the dissipativity-based consensus tracking control (DBCTC) problems of time-varying delayed leader-following nonlinear multiagent systems (LFNMASs) with event-triggered transmission strategy. The switching topologies of the LFNMASs are subject to uncertain and partially unknown generally Markovian jumping process. The control inputs of the following agents are updated according to the proposed event-triggered transmission strategy, which could reduce the communication burden. Based on the eventtriggered transmission condition and distributed consensus protocol, some dissipativity-based criteria obtained by adopting the delay-product-term Lyapunov-Krasovskii functional (DPTLKF) and higher order polynomial based relaxed inequality (HOPRII) are proposed to guarantee LFNMASs consensus. The validity of the main results is verified by two simulation examples.
\end{abstract}

Index Terms-event-triggered consensus strategy, leaderfollowing nonlinear multiagent systems (LFNMASs), generally uncertain Markovian switching topologies, higher order polynomial based relaxed inequality (HOPRII).

\section{INTRODUCTION}

D Uring the past decade, a great many problems of multiagent systems (MASs), such as tracking control, containment control, consensus analysis, formation control, and deep reinforcement learning were extensively investigated by researchers due to their widespread applications in unmanned aerial vehicle formation control, autonomous underwater vehicles cooperative control, spacecraft attitude coordination, and sensor networks information fusion (see [1]-[22] and the references therein). By utilizing the shared communication network topology and cooperative control theory, a group of

This work was supported by the National Natural Science Foundation of China (61903075,U20A20197), and the Chunhui Plan Cooperative Project of Ministry of Education (LN2019006), and Project of Liaoning Province Science and Technology Program (2019-KF-03-02), and the Fundamental Research Funds for the Central Universities (N2026003,N2126006).

J. Wang is with Faculty of Robot Science and Engineering, and School of Future Technologies, Northeastern University, Shenyang 110819, China. H. Zhang is with College of Information Science and Engineering, Northeastern University, Shenyang 110819, China. J. Fu is with State Key Laboratory of Synthetical Automation for Process Industries, Faculty of Robot Science and Engineering, and School of Future Technologies, Northeastern University, Shenyang 110819, China. H. Liang is with the School of Automation Engineering, University of Electronic Science and Technology of China, Chengdu 611731, China. Q. Meng is with Department of Computer Science, Loughborough University, Loughborough LE11 3TU, UK. (email: wjyi168@126.com; hgzhang@ieee.org; junfu@ mail.neu.edu.cn; lianghongjing@uestc.edu.cn; Q.Meng@lboro.ac.uk). agents could carry out difficult tasks with high quality and efficiency [23], [24]. Hence, the cooperative control issue of MASs becomes a research hotspot. The neuro-adaptive cooperative tracking control with prescribed performance of unknown higher-order nonlinear MASs was investigated in [25]. Considering switching directed topologies, the hierarchical cooperative control was investigated for a two-layer networked MASs in [26]. After that, based on sampleddata setting, the research progresses of distributed cooperative control of MASs were addressed in [27]. Based on [27], the investigation of fault tolerant cooperative control in MASs was presented in [28]. For the cooperative control of leaderfollowing MASs, the leader usually has an impact on the movement of the followers. Compared with leaderless MASs, leader-following formation could help the communication and orientation of the team. Hence, the consensus problems of leader-following MASs were developed in recent years (see [3], [24], [29]-[35] and the references therein).

The communication resources in the network environment are usually limited due to channel bandwidth and capacity. Hence, much effort has been made to design energysaving control strategies to reduce the communication and calculation burden caused by the continuous communication [8], which could save network energy consumption. Compared with the time-triggered method, the key advantage of the event-triggered strategy is that the measurement transmission and control tasks are executed only at the violation of eventtriggered condition. Hence, event-triggered data transmission has become popular for its great benefits in saving communication resources [36]. The distributed event-triggered control problem of linear MASs was investigated in [37]. The novel event-triggered control schemes were addressed for the MASs with general linear dynamics in [38]. Based on [38], the leader-following event-triggered consensus control of MASs with different topology structures was investigated in [39]. The distributed event-based consensus of linear MASs with jointly connected switching topologies was investigated in [40]. However, due to the speed of data transmission and the limited bandwidth resources of channels, the communication delay commonly exists in the networks and is a non-negligible element in the consensus problem of MASs [41]. The eventtriggered consensus problem of linear MASs with time-varying communication delays was investigated in [41]. Some novel 
event-triggered control strategies of linear MASs with timevarying delays were investigated in [3], [41]. To our best knowledge, the event-triggered consensus tracking control issues of NMASs with time-varying delay and Markovain switching topologies have not been studied extensively, which is the first motivation of this article.

Many existing results with respect to event-triggered consensus of MASs usually focused on fixed topologies [15], [39], switching topologies [15], [39], [40], directed topologies [42] and undirected topologies [43]. The new leader-follower relation-invariable persistent formation with various switching topologies was proposed in [21]. Due to environment factors and communication interference, the topology structures of MASs may vary according to time [3], [11], [44]. In order to represent the switching structure, it is always characterised as Markovian jumping topologies. Hence, the consensus issues of MASs with Markovian switching topologies were researched in [3], [44]-[47]. Under most circumstances, it is not easy to get exact values of the transition rates because of the equipment performance influence and external environment uncertainties [48], [49]. However, there are few articles for consensus control of MASs with generally uncertain transition rates. Due to the internal and external environment factors in practical application, it is essential to consider more general consensus tracking control of delayed LFNMASs with generally uncertain Markovian switching topologies, which is the second motivation of this article.

For input-output energy-related feature of control systems, the dissipativity problems have attracted considerable attention in static neural networks [50], singular MASs [51], complex networks [52] and fuzzy systems [53]. In [54], based on sliding mode control strategy, the dissipative consensus control issue was researched for multiagent networks. After that, the dissipativity consensus issues were investigated for singular MASs [51] and fuzzy MASs with switching directed topologies [55]. It is also noted that the common Lyapunov function was constructed to deal with dissipativitybased consensus in [51], [54], [55]. However, the dissipative performance has not been introduced into the consensus tracking control of LFNMASs with generally uncertain Markovian switching topologies and event-triggered strategy (ETS), which is the third motivation of this article.

Motivated by the aforementioned researches and based on ETS, the dissipativity-based consensus tracking issue of delayed NMASs with generally uncertain Markovian switching topologies is addressed in this article, and each agent is generally nonlinear dynamics. In the light of graph theory, augmented Markovian DPTLKF, and dissipativity analysis, the consensus tracking problems are proposed in this article. In addition, the corresponding controllers are obtained from dissipativity-based consensus conditions. The primary contributions are summarised as follows

1) Compared with Markovian switching topologies in [3], [44], [45], [51], the generally uncertain Markovian switching topologies of NMASs are investigated in this article, which is more practical because it is hard to precisely estimate the transition rates due to the costs and the stochastic factors.
2) The DPTLKF and HOPRII with tighter lower bound are adopted in this article to tackle the consensus tracking control issues of delayed NMASs, and more information about time-varying delay and its derivative could be contained in the dissipativity-based consensus conditions.

3) Each agent of the MASs is nonlinear and time-varying system, which is capable of providing more nature representations than the normal linear MASs. In addition, ETS is only required to be computed at sampling instants, which could reduce the communication loads.

The structure of this article is as follows. Some preliminaries and LFNMASs are presented in Section II. In Section III, the novel dissipativity-based consensus conditions of NMASs are proposed based on DPTLKF and ETS. The validity of the proposed results is demonstrated by the simulation examples in Section IV. The summaries are proposed in Section V.

\section{Preliminaries}

\section{A. Notation}

$\mathcal{R}^{p}$ and $\mathcal{R}^{p \times q}$ are $p$ real vector space and $p \times q$ real matrices set, respectively. $\|\cdot\|$ is regarded as Euclidean norm. $\otimes$ represents Kronecker product. $Z^{T}$ is regarded as the transpose of matrix $Z . Z>0$ is regarded as that $Z$ is a real symmetric positive definite matrix. $\mathfrak{L}_{2}\left([0,+\infty), \mathcal{R}^{p}\right)$ is the space of $p$-dimensional square summable vector functions. For real matrix $T,\langle w, T w\rangle_{\sigma}$ represents $\int_{0}^{\sigma} w^{T}(\alpha) T w(\alpha) d \alpha$. $\operatorname{Sym}\{Z\}=Z+Z^{T} . I_{p}$ is the $p$-dimensional identity matrix. For any matrices $Z_{1}, Z_{2}$ and $Z_{3},\left(\begin{array}{cc}Z_{1} & Z_{2} \\ * & Z_{3}\end{array}\right)$ stands for $\left(\begin{array}{cc}Z_{1} & Z_{2} \\ Z_{2}^{T} & Z_{3}\end{array}\right) \cdot \mathcal{E}$ represents the mathematical expectation. $e_{\xi}=\left[\Im_{N n \times(\xi-1) N n}, I_{N n}, \Im_{N n \times(17-\xi) N n}\right](\xi=$ $1,2, \cdots, 17), \quad \hat{e}_{\varrho}=\left[\Im_{N n \times(\varrho-1) N n}, I_{N n}, \Im_{N n \times(18-\varrho) N n}\right]$ $(\varrho=1,2, \cdots, 18)$, where $\Im$ indicates the zero matrix.

\section{B. Algebraic Graph Theory}

$\mathbb{G}=(\mathbb{V}, \mathbb{E}, \mathbb{A})$ is a directed graph between the $N$ followers. Here, $\mathbb{V}=\left\{v_{1}, v_{2}, \cdots, v_{N}\right\}$ denotes a set of followers. $\mathbb{E} \subseteq \mathbb{V} \times \mathbb{V}$ denotes a set of directed edges. $\left(v_{k}, v_{l}\right) \in \mathbb{E}$ denotes that node $v_{l}$ is a neighbor of node $v_{k}$, and node $v_{k}$ could acquire information from node $v_{l} . \mathbb{N}_{k}=\left\{l \mid\left(v_{k}, v_{l}\right) \in \mathbb{E}\right\}$ is the neighbor index set of node $v_{k} . \mathbb{A}=\left(a_{k l}\right)_{N \times N}$ is a weighted adjacency matrix. $a_{k l}>0$ if $\left(v_{k}, v_{l}\right) \in \mathbb{E}$, otherwise $a_{k l}=0$. A path from agent $v_{l}$ to $v_{k}$ is a sequence of edges $\left(v_{k}, v_{k_{1}}\right)$, $\left(v_{k_{1}}, v_{k_{2}}\right), \cdots,\left(v_{k_{l}}, v_{l}\right)$ in the graph $\mathbb{G}$ with distinct nodes $v_{k_{p}}$ $(p=1,2, \cdots, l) . \mathbb{L}=\left(l_{k l}\right)_{N \times N}$ is Laplacian matrix satisfying $l_{k k}=\sum_{l \in \mathbb{N}_{k}} a_{k l}$ and $l_{k l}=-a_{k l}(k \neq l)$.

This article primarily focuses on a directed graph $\tilde{\mathbb{G}}$ including a leader $v_{0}$ and $N$ followers. In addition, $\widetilde{\mathbb{G}}$ comprises $\mathbb{G}, v_{0}$ and directed edges from leader $v_{0}$ to followers. The leader could not acquire the information from the followers, and the leader is a neighbor of part of the followers. $\Gamma=\operatorname{diag}\left\{\gamma_{1}, \gamma_{2}, \cdots, \gamma_{N}\right\}$ is the leader adjacency matrix to describe the graph $\tilde{\mathbb{G}}$, where $\gamma_{k}>0$ if and only if the node $v_{k}$ could acquire the information from $v_{0}$, otherwise $\gamma_{k}=0$. 


\section{Assumptions}

Assumption 1: ( [56]) Each graph $\tilde{\mathbb{G}}$ including the leader and followers has a directed spanning tree with a root of the leader.

Assumption 2: ( [3]) All sampling periods of agents are synchronized by the same clock.

Assumption 3: ( [57]) For the continuous and bounded function $f_{\iota}(x)$, there are real scalars $e_{\iota}^{-}, e_{\iota}^{+}$such that the inequality $e_{\iota}^{-} \leq \frac{f_{\iota}(x)-f_{\iota}(y)}{x-y} \leq e_{\iota}^{+}(\iota=1,2, \cdots, n)$ holds, where $f_{\iota}(0)=0, x, y \in \mathcal{R}, x \neq y$.

\section{Problem Formulation}

$\left\{r_{t}, t \geq 0\right\}$ is a right-continuous Markovian process. Its value is the finite state space $\mathcal{S}=\{1,2, \cdots, \mathcal{M}\}$ with $\Pi=\left(\pi_{i j}\right)(i, j \in \mathcal{S})$.

$$
\operatorname{Pr}\left\{r_{t+\Delta t}=j \mid r_{t}=i\right\}=\left\{\begin{array}{c}
\pi_{i j} \Delta t+o(\Delta t), i \neq j, \\
1+\pi_{i j} \Delta t+o(\Delta t), i=j,
\end{array}\right.
$$

where $\Delta t>0$, and $\lim _{\Delta t \rightarrow 0}(o(\Delta t) / \Delta t)=0 . \pi_{i j} \geq 0(i \neq j)$ is the transition rate from mode $i$ at time $t$ to mode $j$ at time $t+\Delta t$, and $\pi_{i i}=-\sum_{j=1, j \neq i}^{\mathcal{M}} \pi_{i j}$.

The transition rate matrix (TRM) $\Pi$ for generally uncertain Markovian is represented as

$$
\Pi=\left(\begin{array}{ccccc}
\tilde{\pi}_{11}+\Delta_{11} & ? & \tilde{\pi}_{13}+\Delta_{13} & \cdots & ? \\
\tilde{\pi}_{21}+\Delta_{21} & ? & ? & \cdots & ? \\
\vdots & \vdots & \vdots & \ddots & \vdots \\
\tilde{\pi}_{\mathcal{M} 1}+\Delta_{\mathcal{M} 1} & ? & ? & \cdots & ?
\end{array}\right)
$$

where "?" denotes the completely unknown transition rate. $\tilde{\pi}_{i j}$ and $\Delta_{i j}$ denote the estimate value and estimate error of the uncertain transition rate $\pi_{i j}$, respectively. $\left\|\triangle_{i j}\right\| \leq \varpi_{i j}$ and $\varpi_{i j} \geq 0 . \tilde{\pi}_{i j}$ and $\varpi_{i j}$ are known values. For $\forall i \in \mathcal{S}$, we denote $\mathcal{S}^{i}=\mathcal{S}_{\mathrm{k}}^{i} \bigcup \mathcal{S}_{\mathrm{uk}}^{i}$, where $\mathcal{S}_{\mathrm{k}}^{i}=\{j$ : The estimate value of $\pi_{i j}$ is known for $\left.j \in \mathcal{S}\right\}$ and $\mathcal{S}_{\mathrm{uk}}^{i}=\{j$ : The estimate value of $\pi_{i j}$ is unknown for $\left.j \in \mathcal{S}\right\}$.

On the basis of the characteristics of the transition rates, the following cases are assumed.

If $\mathcal{S}_{\mathrm{k}}^{i} \neq \mathcal{S}$ and $i \notin \mathcal{S}_{\mathrm{k}}^{i}$, then $\tilde{\pi}_{i j}-\varpi_{i j} \geq 0,\left(\forall j \in \mathcal{S}_{\mathrm{k}}^{i}\right)$.

If $\mathcal{S}_{\mathrm{k}}^{i} \neq \mathcal{S}$ and $i \in \mathcal{S}_{\mathrm{k}}^{i}$, then $\tilde{\pi}_{i j}-\varpi_{i j} \geq 0,\left(\forall j \in \mathcal{S}_{\mathrm{k}}^{i}, j \neq i\right)$, $\tilde{\pi}_{i i}+\varpi_{i i} \leq 0$, and $\sum_{j \in \mathcal{S}_{\mathbf{k}}^{i}} \tilde{\pi}_{i j} \leq 0$.

If $\mathcal{S}_{\mathrm{k}}^{i}=\mathcal{S}$, then $\tilde{\pi}_{i j}-\varpi_{i j} \geq 0,(\forall j \in \mathcal{S}, j \neq i), \tilde{\pi}_{i i}=$ $-\sum_{j=1, j \neq i} \tilde{\pi}_{i j} \leq 0$, and $\varpi_{i i}=\sum_{j=1, j \neq i} \varpi_{i j} \geq 0$.

Remark 1: Different from most existing TRM, the generally uncertain TRM (1) including both bounded uncertain and partially unknown elements is considered for NMASs in this article, which is more general and applicable. When $\Delta_{i j}=0$, generally uncertain TRM (1) is simplified as a special case of partially unknown TRM.

The following NMASs with $N$ following agents are considered in this article. The dynamics of the $k$ th agent are given as

$$
\left\{\begin{array}{c}
\dot{x}_{k}(t)=A x_{k}(t)+B f\left(x_{k}(t)\right)+C f\left(x_{k}(t-\varphi(t))\right. \\
+u_{k}(t)+B_{w} w_{k}(t) \\
\psi_{k}(t)=D x_{k}(t), k=1,2, \cdots, N
\end{array}\right.
$$

where $x_{k}(t) \in \mathcal{R}^{n}, u_{k}(t) \in \mathcal{R}^{n}$ and $\psi_{k}(t) \in \mathcal{R}^{n}$ are the state, control input and output signal of the $k$ th agent, respectively. $w_{k}(t) \in \mathfrak{L}_{2}\left([0,+\infty), \mathcal{R}^{n}\right)$ denotes the exogenous disturbance. $A \in \mathcal{R}^{n \times n}, B \in \mathcal{R}^{n \times n}, C \in \mathcal{R}^{n \times n}, D \in \mathcal{R}^{n \times n}$ and $B_{w} \in$ $\mathcal{R}^{n \times n}$ are constant matrices. $f\left(x_{k}(t)\right): \mathcal{R}^{n} \times[0,+\infty) \rightarrow \mathcal{R}^{n}$ is the nonlinear continuous differentiable function of $k t h$ agent system and satisfies Assumption 3. The time-varying delay $\varphi(t)$ satisfies $0 \leq \varphi(t) \leq \varphi, \mu_{1} \leq \dot{\varphi}(t) \leq \mu_{2}$, where $\varphi, \mu_{1}$ and $\mu_{2}$ are constants.

The initial situation of system (2) is given as follows. $x_{k}(s)=\phi_{k 0}(s) \in \mathcal{C}\left([-\varphi, 0], \mathcal{R}^{n}\right)(k=1,2, \cdots, N)$, and $\mathcal{C}\left([-\varphi, 0], \mathcal{R}^{n}\right)$ denotes the group of continuous functions from $[-\varphi, 0]$ to $\mathcal{R}^{n}$.

The dynamics of the leader are described as

$$
\left\{\begin{array}{c}
\dot{x}_{0}(t)=A x_{0}(t)+B f\left(x_{0}(t)\right)+C f\left(x_{0}(t-\varphi(t))\right), \\
\psi_{0}(t)=D x_{0}(t),
\end{array}\right.
$$

where $x_{0}(t) \in \mathcal{R}^{n}$ and $\psi_{0}(t) \in \mathcal{R}^{n}$ represent state and output of the leader.

To save network communication resources and make the NMASs reach consensus, a distributed event-triggered strategy is equipped with each agent. If the event-triggered strategy is satisfied, the agent could receive the sampled data from its neighbors.

$$
\begin{aligned}
& {\left[y_{k}\left(t_{p}^{k}+\beta h\right)\right]^{T} \Omega y_{k}\left(t_{p}^{k}+\beta h\right)} \\
& \leq \theta_{k}\left[\omega_{k}\left(t_{p}^{k}+\beta h\right)\right]^{T} \Omega \omega_{k}\left(t_{p}^{k}+\beta h\right),
\end{aligned}
$$

where $\theta_{k}>0$ means the threshold parameter. $\Omega$ means the event-triggered matrix, which satisfies $\Omega \in \mathcal{R}^{n \times n}$ and $\Omega>0$. $h$ denotes the sampling period, and $t_{p}^{k}$ denotes the $p$ th eventtriggered instant of the agent $k . t_{p}^{k}+\beta h$ represents the currently sampled instant and $y_{k}\left(t_{p}^{k}+\beta h\right)=x_{k}\left(t_{p}^{k}\right)-x_{k}\left(t_{p}^{k}+\beta h\right)$, $\omega_{k}\left(t_{p}^{k}+\beta h\right)=\sum_{l \in \mathbb{N}_{k}} a_{k l}\left[x_{k}\left(t_{p}^{k}\right)-x_{l}\left(t_{\hat{p}}^{l}\right)\right]+\gamma_{k}\left[x_{k}\left(t_{p}^{k}\right)-x_{0}\left(t_{p}^{k}+\right.\right.$ $\beta h)], t_{\hat{p}}^{l}=\max \left\{t \mid t \in\left\{t_{p}^{l}, p=1,2,3, \cdots\right\}, t \leq t_{p}^{l}+\beta h\right\}$.

Remark 2: Since the event-triggered time sequence $\left\{t_{0}^{k}, t_{1}^{k}, t_{2}^{k}, \cdots\right\}$ is contained in sampling time sequence $\{0, h, 2 h, \cdots\}$, the minimum event interval time $\left\{t_{p+1}^{k}-t_{p}^{k}\right\}$ for $\forall k$ is greater than or equal to the sampling period $h$. Thus, Zeno behavior doesn't happen.

Consider the LFMASs consensus protocol

$$
\begin{aligned}
& u_{k}(t)=-K\left(r_{t}\right)\left\{\sum_{l \in \mathbb{N}_{k}} a_{k l}\left(r_{t}\right)\left[x_{k}\left(t_{p}^{k}\right)-x_{l}\left(t_{\hat{p}}^{l}\right)\right]\right. \\
& \left.+\gamma_{k}\left(r_{t}\right)\left[x_{k}\left(t_{p}^{k}\right)-x_{0}(q h)\right]\right\} \quad k=1,2, \cdots, N,
\end{aligned}
$$

where $t \in\left[t_{p}^{k}, t_{p+1}^{k}\right) \bigcap[q h,(q+1) h)$, and $q$ denotes an integral. $K\left(r_{t}\right)$ is the feedback gain matrix. $a_{k l}\left(r_{t}\right)$ is the element of the weighted adjacency $\mathbb{A}\left(r_{t}\right) . \gamma_{k}\left(r_{t}\right)$ is the element of leader adjacency matrix. $\gamma_{k}\left(r_{t}\right)>0$ if $k t h$ agent could receive information from the leader, otherwise $\gamma_{k}\left(r_{t}\right)=0$.

Remark 3: The ETS (4) is adopted to decrease needless communication. In addition, the generally uncertain Markovian switching topologies in this article including the uncertain and unknown transition process are more general than the Markovian switching topologies with completely known transition rates. 
Define the next transmission instant $t_{p+1}^{k}$ for the $k$ th agent

$$
\begin{aligned}
& t_{p+1}^{k}=t_{p}^{k}+\min _{\beta \geq 1}\left\{\beta h \mid y_{k}^{T}\left(t_{p}^{k}+\beta h\right) \Omega y_{k}\left(t_{p}^{k}+\beta h\right)\right. \\
& \left.>\theta_{k} \omega_{k}^{T}\left(t_{p}^{k}+\beta h\right) \Omega \omega_{k}\left(t_{p}^{k}+\beta h\right)\right\},
\end{aligned}
$$

where $\left[t_{p}^{k}, t_{p+1}^{k}\right)=\bigcup_{q h=t_{p}^{k}}^{t_{p+1}^{k}-h}[q h,(q+1) h)$. Let $y_{k}(q h)=$ $x_{k}\left(t_{p}^{k}\right)-x_{k}(q h), \beta h=q h-x_{p}^{k}$ and $v_{k}(q h)=x_{k}(q h)-x_{0}(q h)$, for $t \in[q h,(q+1) h)$, hence the consensus protocol is rewritten as

$$
\begin{aligned}
& u_{k}(t)=-K\left(r_{t}\right)\left\{\sum _ { l \in \mathbb { N } _ { k } } a _ { k l } ( r _ { t } ) \left[y_{k}(q h)-y_{l}(q h)+v_{k}(q h)\right.\right. \\
& \left.\left.-v_{l}(q h)\right]+\gamma_{k}\left(r_{t}\right)\left[y_{k}(q h)+v_{k}(q h)\right]\right\}, k=1,2, \cdots, N .
\end{aligned}
$$

According to (2), (3) and (7), one obtains the following error dynamics system

$$
\left\{\begin{array}{c}
\dot{v}_{k}(t)=A v_{k}(t)+B g\left(v_{k}(t)\right)+C g\left(v_{k}(t-\varphi(t))\right) \\
-K\left(r_{t}\right)\left\{\sum _ { l \in \mathbb { N } _ { k } } a _ { k l } ( r _ { t } ) \left[y_{k}(q h)-y_{l}(q h)+v_{k}(q h)\right.\right. \\
\left.\left.-v_{l}(q h)\right]+\gamma_{k}\left(r_{t}\right)\left[y_{k}(q h)+v_{k}(q h)\right]\right\}+B_{w} w_{k}(t), \\
h_{k}(t)=D v_{k}(t), t \in[q h,(q+1) h), k=1,2, \cdots, N .
\end{array}\right.
$$

where $v_{k}(t)=x_{k}(t)-x_{0}(t), g\left(v_{k}(t)\right)=f\left(x_{k}(t)\right)-f\left(x_{0}(t)\right)$, $h_{k}(t)=\psi_{k}(t)-\psi_{0}(t), g\left(v_{k}(t-\varphi(t))\right)=f\left(x_{k}(t-\varphi(t))\right)-$ $f\left(x_{0}(t-\varphi(t))\right)$. The structure of the control system for error system (8) is given in Fig. 1.

Assume $v(t)=\left[v_{1}^{T}(t), v_{2}^{T}(t), \cdots, v_{N}^{T}(t)\right]^{T}$, $y(t)=\left[y_{1}^{T}(t), y_{2}^{T}(t), \cdots, y_{N}^{T}(t)\right]^{T}$, $h(t)=\left[h_{1}^{T}(t), h_{2}^{T}(t), \cdots, h_{N}^{T}(t)\right]^{T}$, $w(t)=\left[w_{1}^{T}(t), w_{2}^{T}(t), \cdots, w_{N}^{T}(t)\right]^{T}$, $g(v(t))=\left[g^{T}\left(v_{1}(t)\right), g^{T}\left(v_{2}(t)\right), \cdots, g^{T}\left(v_{N}(t)\right)\right]^{T}$, $G_{i}=\mathbb{L}_{i}+\Gamma_{i}, \Gamma_{i}=\operatorname{diag}\left\{\gamma_{1, i}, \gamma_{2, i}, \cdots, \gamma_{N, i}\right\}$. Each value of $r_{t}$ is represented by $i(i \in \mathcal{S})$. According to (8), one obtains

$$
\left\{\begin{array}{c}
\dot{v}(t)=\left(I_{N} \otimes A\right) v(t)+\left(I_{N} \otimes B\right) g(v(t)) \\
+\left(I_{N} \otimes C\right) g(v(t-\varphi(t)))-\left(G_{i} \otimes K_{i}\right) v(t-d(t)) \\
-\left(G_{i} \otimes K_{i}\right) y(t-d(t))+\left(I_{N} \otimes B_{w}\right) w(t), \\
h(t)=\left(I_{N} \otimes D\right) v(t), t \in[q h,(q+1) h),
\end{array}\right.
$$

where $t-d(t)=q h, 0 \leq d(t)=t-q h<h$, and $d(t)$ denotes piecewise-linear with $\dot{d}(t)=1, t \neq q h$.

The initial condition of state $v(s)$ is given as follows $v(0)=\left[v_{1}^{T}(0), v_{2}^{T}(0), \cdots, v_{N}^{T}(0)\right]^{T}$.

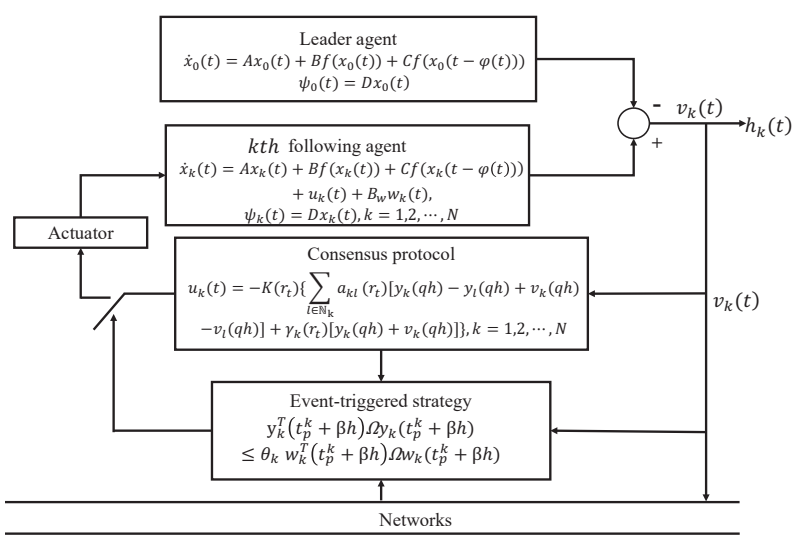

Fig. 1 Structure of the control system.
Definition 1: Under generally uncertain Markovian switching topologies, the leader-following consensus in NMASs (2) and (3) with $w_{k}(t)=0$ is achieved if

$$
\lim _{t \rightarrow \infty} \mathcal{E}\left\|x_{k}(t)-x_{0}(t)\right\|=0, k=1,2, \cdots, N
$$

holds for any initial conditions $\phi_{k 0}(s) \in \mathcal{C}\left([-\varphi, 0], \mathcal{R}^{n}\right)(k=$ $1,2, \cdots, N)$.

Definition 2: For NMASs (2) and (3), the consensus tracking problem could be tackled by adopting ETS (4) and consensus protocol (5) if the following conditions are satisfied.

1) Under the condition $w_{k}(t)=0$, the nonlinear multiagent error system (9) is leader-following consensus.

2) With zero initial condition, a scalar $\rho>0$, the real symmetric matrices $\mathcal{P} \leq 0, \mathcal{R}$ and real matrix $\mathcal{T}$, the nonlinear multiagent error system $(9)$ is strictly $(\mathcal{P}, \mathcal{T}, \mathcal{R})-\rho$ dissipative if the following inequality holds for any $\sigma \geq 0$

$$
\langle h, \mathcal{P} h\rangle_{\sigma}+2\langle h, \mathcal{T} w\rangle_{\sigma}+\langle w, \mathcal{R} w\rangle_{\sigma} \geq \rho\langle w, w\rangle_{\sigma} .
$$

Lemma 1: (see [58]). For $v(t) \in W\left[\kappa_{1}, \kappa_{2}\right), v\left(\kappa_{1}\right)=0$ and any matrix $Z>0$, the following inequality holds

$$
\frac{\pi^{2}}{4} \int_{\kappa_{1}}^{\kappa_{2}} v^{T}(s) Z v(s) d s \leq\left(\kappa_{2}-\kappa_{1}\right)^{2} \int_{\kappa_{1}}^{\kappa_{2}} \dot{v}^{T}(s) Z \dot{v}(s) d s .
$$

Lemma 2: (HOPRII, [59]). $x(t)$ is a differentiable function in $\left[a_{1}, a_{2}\right] \rightarrow \mathcal{R}^{n}$ for a time-varying scalar $a(t) \in\left[a_{1}, a_{2}\right]$. For symmetric matrices $Z_{l}>0(l=1,2)$, any matrices $N_{l}>0(l=1,2)$, the following inequality holds.

$$
\begin{aligned}
& a_{12} \int_{a_{1}}^{a(t)} \dot{x}^{T}(s) Z_{1} \dot{x}(s) d s+a_{12} \int_{a(t)}^{a_{2}} \dot{x}^{T}(s) Z_{2} \dot{x}(s) d s \geq \\
& \zeta^{T}(t)\left[\begin{array}{l}
\omega_{1} \\
\omega_{2}
\end{array}\right]^{T} \Delta\left[\begin{array}{l}
\omega_{1} \\
\omega_{2}
\end{array}\right] \zeta(t),
\end{aligned}
$$

where $\Delta=\left[\begin{array}{cc}\widetilde{Z_{1}}+(1-\gamma) \widetilde{\mho}_{1} & (1-\gamma) N_{1}+\gamma N_{2} \\ * & \widetilde{Z}_{2}+\gamma \widetilde{\mho}_{2}\end{array}\right]$, $\gamma=\frac{a(t)-a_{1}}{a_{12}}, a_{12}=a_{2}-a_{1}$, $\zeta(t)=\left[x^{T}\left(a_{2}\right), x^{T}(a(t)), x^{T}\left(a_{1}\right), \zeta_{1}^{T}(t), \quad \zeta_{2}^{T}(t), \quad \zeta_{3}^{T}(t)\right.$, $\left.\zeta_{4}^{T}(t), \zeta_{5}^{T}(t), \zeta_{6}^{T}(t)\right]^{T}$,

$\zeta_{1}(t)=\frac{1}{a(t)-a_{1}} \int_{a_{1}}^{a(t)} x(s) d s$,

$\zeta_{2}(t)=\frac{1}{a_{2}-a(t)} \int_{a(t)}^{a_{2}} x(s) d s$,

$\zeta_{3}(t)=\frac{2}{\left(a(t)-a_{1}\right)^{2}} \int_{a_{1}}^{a(t)} \int_{u}^{a(t)} x(s) d s d u$,

$\zeta_{4}(t)=\frac{2}{\left(a_{2}-a(t)\right)^{2}} \int_{a(t)}^{a_{2}} \int_{u}^{a_{2}} x(s) d s d u$,

$\zeta_{5}(t)=\frac{6}{\left(a(t)-a_{1}\right)^{3}} \int_{a_{1}}^{a(t)} \int_{u}^{a(t)} \int_{v}^{a(t)} x(s) d s d v d u$,

$\zeta_{6}(t)=\frac{6}{\left(a_{2}-a(t)\right)^{3}} \int_{a(t)}^{a_{2}} \int_{u}^{a_{2}} \int_{v}^{a_{2}} x(s) d s d v d u$,

$\widetilde{Z}_{1}=\operatorname{diag}\left\{Z_{1}, 3 Z_{1}, 5 Z_{1}, 7 Z_{1}\right\}$,

$\widetilde{Z}_{2}=\operatorname{diag}\left\{Z_{2}, 3 Z_{2}, 5 Z_{2}, 7 Z_{2}\right\}$,

$\widetilde{\mho}_{1}=\widetilde{Z}_{1}-N_{2} \widetilde{Z}_{2}^{-1} N_{2}^{T}, \widetilde{\mho}_{2}=\widetilde{Z}_{2}-N_{1}^{T} \widetilde{Z}_{1}^{-1} N_{1}$,

$\omega_{1}=\left[\left(\widetilde{e}_{2}-\widetilde{e}_{3}\right)^{T},\left(\widetilde{e}_{2}+\widetilde{e}_{3}-2 \widetilde{e}_{4}\right)^{T},\left(\widetilde{e}_{2}-\widetilde{e}_{3}+6 \widetilde{e}_{4}-6 \widetilde{e}_{6}\right)^{T}\right.$, $\left.\left(\widetilde{e}_{2}+\widetilde{e}_{3}-12 \widetilde{e}_{4}+30 \widetilde{e}_{6}-20 \widetilde{e}_{8}\right)^{T}\right]^{T}$,

$\omega_{2}=\left[\left(\widetilde{e}_{1}-\widetilde{e}_{2}\right)^{T},\left(\widetilde{e}_{1}+\widetilde{e}_{2}-2 \widetilde{e}_{5}\right)^{T},\left(\widetilde{e}_{1}-\widetilde{e}_{2}+6 \widetilde{e}_{5}-6 \widetilde{e}_{7}\right)^{T}\right.$, $\left.\left(\widetilde{e}_{1}+\widetilde{e}_{2}-12 \widetilde{e}_{5}+30 \widetilde{e}_{7}-20 \widetilde{e}_{9}\right)^{T}\right]^{T}$,

$\widetilde{e}_{k}=\left[0_{n \times(k-1) n} I_{n \times n} 0_{n \times(9-k) n}\right],(k=1,2, \cdots, 9)$.

Remark 4: According to [59], Lemma 3 provides a tighter lower bound, which is close to the integral term in 
(12). In addition, there is no positive requirement for matrix $\Delta$, which could provide with extra freedom.

\section{MAIN RESUltS}

In this part, with $w_{k}(t)=0$, the consensus issue of error system (9) is introduced firstly. After that, based on strict $(\mathcal{P}, \mathcal{T}, \mathcal{R})-\rho$-dissipativity theory, the dissipativity-based consensus tracking problem is investigated by protocol (5).

\section{A. Consensus Analysis}

In this section, the tracking consensus of NMASs (2) and (3) with $w_{k}(t)=0$ is obtained based on DPTLKF and consensus protocol.

Theorem 1 Under Assumptions 1-3, scaler $m>0$, the consensus tracking control of NMASs (2) and (3) with $w_{k}(t)=0$ is achieved if there are any matrices $X_{1} \in \mathcal{R}^{2 N n \times 2 N n}$, $X_{2} \in \mathcal{R}^{6 N n \times 6 N n}, Y_{l} \in \mathcal{R}^{4 N n \times 4 N n}, J_{i} \in \mathcal{R}^{N n \times N n}$, invertible matrix $F \in \mathcal{R}^{N n \times N n}$, symmetric matrices $U_{1} \in$ $\mathcal{R}^{4 N n \times 4 N n}, U_{\nu} \in \mathcal{R}^{2 n \times 2 n}, U_{\nu}>0(\nu=2,3), Q_{k p}$, $Q_{k q} \in \mathcal{R}^{2 N n \times 2 N n}(k=3,4), Z_{l} \in \mathcal{R}^{N n \times N n}(l=1,2)$, symmetric positive definite matrices $P_{i} \in \mathcal{R}^{N n \times N n}, V_{i j} \in$ $\mathcal{R}^{N n \times N n}, R \in \mathcal{R}^{N n \times N n}, M_{\nu} \in \mathcal{R}^{N n \times N n}(\nu=2,3), M_{1}$ $\in \mathcal{R}^{2 N n \times 2 N n}, Q_{l} \in \mathcal{R}^{2 N n \times 2 N n}(l=1,2)$, and diagonal matrices $R_{\alpha_{1}}>0\left(\alpha_{1}=1,2,3\right)$, such that for any $i \in \mathcal{S}$, the following linear matrix inequalities (LMIs) are satisfied.

If $i \notin S_{\mathrm{k}}^{i}$,

$$
\begin{aligned}
& P_{j}-P_{i}-V_{i j} \leq 0\left(\forall j \in S_{\mathrm{k}}^{i}\right), \\
& P_{j}-P_{i} \leq 0\left(\forall j \in S_{\mathrm{uk}}^{i}, j \neq i\right), \\
& {\left[\begin{array}{cc}
\Phi\left(\mu_{1}, 0\right) & w_{1}^{T} Y_{2} \\
* & -\widehat{M}_{2}
\end{array}\right]<0} \\
& {\left[\begin{array}{cc}
\Phi\left(\mu_{1}, \varphi\right) & w_{2}^{T} Y_{1}^{T} \\
* & -\widehat{M}_{2}
\end{array}\right]<0,} \\
& {\left[\begin{array}{cc}
\Phi\left(\mu_{2}, 0\right) & w_{1}^{T} Y_{2} \\
* & -\widehat{M}_{2}
\end{array}\right]<0,} \\
& {\left[\begin{array}{cc}
\Phi\left(\mu_{2}, \varphi\right) & w_{2}^{T} Y_{1}^{T} \\
* & -\widehat{M}_{2}
\end{array}\right]<0,} \\
& {\left[\begin{array}{cc}
U_{2} & X_{1} \\
* & U_{3}
\end{array}\right] \geq 0,\left[\begin{array}{cc}
\hat{Q}_{3 q} & X_{2} \\
* & \hat{Q}_{4 q}
\end{array}\right] \geq 0,}
\end{aligned}
$$

$\zeta_{0}>0, \zeta_{1}>0, Q_{k, l}>0, N_{\rho, l}>0(k=3,4, \rho, l=1,2)$,

where

$\Phi\left(\mu_{1}, 0\right)=\left.\Xi(\dot{\varphi}(t), \varphi(t))\right|_{\dot{\varphi}(t)=\mu_{1}, \varphi(t)=0}+\Xi_{t}+\Xi_{i i}$,

$\Phi\left(\mu_{1}, \varphi\right)=\left.\Xi(\dot{\varphi}(t), \varphi(t))\right|_{\dot{\varphi}(t)=\mu_{1}, \varphi(t)=\varphi}+\Xi_{t}+\Xi_{i i}$,

$\Phi\left(\mu_{2}, 0\right)=\left.\Xi(\dot{\varphi}(t), \varphi(t))\right|_{\dot{\varphi}(t)=\mu_{2}, \varphi(t)=0}+\Xi_{t}+\Xi_{i i}$,

$\Phi\left(\mu_{2}, \varphi\right)=\left.\Xi(\dot{\varphi}(t), \varphi(t))\right|_{\dot{\varphi}(t)=\mu_{2}, \varphi(t)=\varphi}+\Xi_{t}+\Xi_{i i}$,

$\Xi(\dot{\varphi}(t), \varphi(t))=\dot{\varphi}(t) \eta_{2}^{T} Q_{1} \eta_{2}+\operatorname{Sym}\left\{\left[\varphi(t) e_{7}^{T}+(\varphi-\varphi(t)) e_{8}^{T}\right]\right.$

$\left.\times R\left(e_{1}-e_{3}\right)\right\}+\dot{\varphi}(t)\left[\Omega_{3}^{T} U_{2} \Omega_{3}-\Omega_{4}^{T} U_{3} \Omega_{4}\right]+\operatorname{Sym}\left\{\Omega_{1}^{T} U_{1} \Omega_{2}\right\}$

$+\operatorname{Sym}\left\{\Omega_{3}^{T} U_{2} \Omega_{5}\right\}+\operatorname{Sym}\left\{\Omega_{4}^{T} U_{3} \Omega_{6}\right\}+\Omega_{11}^{T}\left(Q_{3 p}-\varphi(t) Q_{3 q}\right)$

$\Omega_{11}-(1-\dot{\varphi}(t)) \Omega_{12}^{T}\left(Q_{3 p}-\varphi(t) Q_{3 q}\right) \Omega_{12}+(1-\dot{\varphi}(t)) \Omega_{12}^{T}\left(Q_{4 p}\right.$

$\left.+(\varphi-\varphi(t)) Q_{4 q}\right) \Omega_{12}-\Omega_{13}^{T}\left(Q_{4 p}+(\varphi-\varphi(t)) Q_{4 q}\right) \Omega_{13}$

$-\operatorname{Sym}\left\{\Omega_{7}^{T} \hat{Q}_{3 q} \Omega_{8}+\Omega_{9}^{T} \hat{Q}_{4 q} \Omega_{10}\right\}-\delta \varphi \Omega_{8}^{T} \hat{Q}_{3 q} \Omega_{8}$ $-(1-\delta) \varphi \Omega_{10}^{T} \hat{Q}_{4 q} \Omega_{10}-\frac{1}{\varphi}\left[\begin{array}{c}\Omega_{7} \\ \Omega_{9}\end{array}\right]^{T}\left[\begin{array}{cc}\hat{Q}_{3 q} & X_{2} \\ * & \hat{Q}_{4 q}\end{array}\right]\left[\begin{array}{c}\Omega_{7} \\ \Omega_{9}\end{array}\right]$ $-\left[\begin{array}{l}w_{1} \\ w_{2}\end{array}\right]^{T} \hat{\phi}_{2}(\delta, 1-\delta)\left[\begin{array}{l}w_{1} \\ w_{2}\end{array}\right], \Xi_{t}=\operatorname{Sym}\left\{e_{1}^{T} P_{i} e_{13}\right\}+\eta_{1}^{T} Q_{1} \eta_{1}-$ $\eta_{2}^{T} Q_{1} \eta_{2}+\eta_{1}^{T} Q_{2} \eta_{1}-\eta_{3}^{T} Q_{2} \eta_{3}+\varphi \Omega_{11}^{T} M_{1} \Omega_{11}+\varphi^{2} e_{13}^{T} M_{2} e_{13}+$ $h^{2} e_{13}^{T} M_{3} e_{13}-\frac{\pi^{2}}{4}\left(e_{1}-e_{16}\right)^{T} M_{3}\left(e_{1}-e_{16}\right)+e_{1}^{T} Z_{1} e_{1}-$ $e_{2}^{T} Z_{1} e_{2}+e_{2}^{T} Z_{2} e_{2}-e_{3}^{T} Z_{2} e_{3}+\operatorname{Sym}\left\{-e_{1}^{T}\left(I_{N} \otimes F\right) e_{13}+e_{1}^{T}\left(I_{N} \otimes\right.\right.$ $F A) e_{1}+e_{1}^{T}\left(I_{N} \otimes F B\right) e_{4}+e_{1}^{T}\left(I_{N} \otimes F C\right) e_{5}-e_{1}^{T}\left(G_{i} \otimes J_{i}\right) e_{16}-$ $\left.e_{1}^{T}\left(G_{i} \otimes J_{i}\right) e_{17}\right\}+\operatorname{Sym}\left\{-m e_{13}^{T}\left(I_{N} \otimes F\right) e_{13}+m e_{13}^{T}\left(I_{N} \otimes\right.\right.$ $F A) e_{1}+m e_{13}^{T}\left(I_{N} \otimes F B\right) e_{4}+m e_{13}^{T}\left(I_{N} \otimes F C\right) e_{5}-m e_{13}^{T}\left(G_{i} \otimes\right.$ $\left.\left.J_{i}\right) e_{16}-m e_{13}^{T}\left(G_{i} \otimes J_{i}\right) e_{17}\right\}+\left(e_{16}+e_{17}\right)^{T}\left(G_{i}^{T} \Theta G_{i} \otimes \Omega\right)\left(e_{16}+\right.$ $\left.e_{17}\right)-e_{17}^{T}\left(I_{N} \otimes \Omega\right) e_{17}+\eta_{1}^{T} \Xi_{1} \eta_{1}+\eta_{2}^{T} \Xi_{2} \eta_{2}+\eta_{3}^{T} \Xi_{3} \eta_{3}$,

$\Xi_{i i}=e_{1}^{T} \sum_{j \in S_{\mathrm{k}}^{i}}^{\mathcal{M}}\left[\underline{\pi}_{i j}\left(P_{j}-P_{i}\right)+2 \varpi_{i j} V_{i j}\right] e_{1}, \zeta_{0}=\left.\zeta_{\delta}\right|_{\delta=0}$,

$\zeta_{1}=\left.\zeta_{\delta}\right|_{\delta=1}, \quad \zeta_{\delta}=U_{1}+\varphi \Lambda_{1}^{T}\left(\delta U_{2}+\right.$ $\left.(1-\delta) U_{3}\right) \Lambda_{1}+\operatorname{Sym}\left\{\Lambda_{1}^{T} U_{2} \Lambda_{2}+\Lambda_{1}^{T} U_{3} \Lambda_{3}\right\}+$ $\frac{1}{\varphi}\left[\begin{array}{c}\Lambda_{2} \\ \Lambda_{3}\end{array}\right]^{T}\left[\begin{array}{cc}U_{2} & X_{1} \\ * & U_{3}\end{array}\right]\left[\begin{array}{c}\Lambda_{2} \\ \Lambda_{3}\end{array}\right]$

$\Lambda_{1}=\left[\begin{array}{ll}\tilde{e}_{1}^{T} & 0\end{array}\right]^{T}, \Lambda_{2}=\left[\begin{array}{ll}0 & \tilde{e}_{3}^{T}\end{array}\right]^{T}, \Lambda_{3}=\left[\begin{array}{ll}0 & \tilde{e}_{4}^{T}\end{array}\right]^{T}$,

$\tilde{e}_{\alpha}=\left[0_{N n \times(\alpha-1) N n} I_{N n \times N n} 0_{N n \times(4-\alpha) N n}\right], \alpha=1,2,3,4$.

$\eta_{1}=\left[e_{1}^{T}, e_{4}^{T}\right], \eta_{2}=\left[e_{2}^{T}, e_{5}^{T}\right], \eta_{3}=\left[e_{3}^{T}, e_{6}^{T}\right], \delta=\frac{\varphi(t)}{\varphi}$,

$\hat{\phi}_{2}(\delta, 1-\delta)=\left[\begin{array}{cc}(2-\delta) \widehat{M}_{2} & (1-\delta) Y_{1}+\delta Y_{2} \\ * & (1+\delta) \widehat{M}_{2}\end{array}\right]$,

$\widehat{M}_{2}=\operatorname{diag}\left\{M_{2}, 3 M_{2}, 5 M_{2}, 7 M_{2}\right\}$,

$\hat{\Theta}_{i}=\varpi_{i i}-\sum_{j \in S_{k}^{i}, j \neq i}^{\mathcal{M}} \varpi_{i j}, \underline{\pi}_{i}=-\bar{\pi}_{i i}-\sum_{j \in S_{k}^{i}, j \neq i}^{\mathcal{M}} \underline{\pi}_{i j}$,

$\underline{\pi}_{i j}=\tilde{\pi}_{i j}-\varpi_{i j}, \bar{\pi}_{i j}=\tilde{\pi}_{i j}+\varpi_{i j}$,

$\Omega_{1}=\left[e_{1}^{T}, e_{2}^{T}, \varphi(t) e_{7}^{T},(\varphi-\varphi(t)) e_{8}^{T}\right]^{T}$,

$\Omega_{2}=\left[e_{13}^{T},(1-\dot{\varphi}(t)) e_{14}^{T}, e_{1}^{T}-(1-\dot{\varphi}(t)) e_{2}^{T},(1-\dot{\varphi}(t)) e_{2}^{T}-\right.$

$\left.e_{3}^{T}\right]^{T}, \Omega_{3}=\left[e_{1}^{T}, e_{7}^{T}\right]^{T}, \Omega_{4}=\left[e_{1}^{T}, e_{8}^{T}\right]^{T}$,

$\Omega_{5}=\left[\varphi(t) e_{13}^{T}, e_{1}^{T}-(1-\dot{\varphi}(t)) e_{2}^{T}-\dot{\varphi}(t) e_{7}^{T}\right]^{T}$,

$\Omega_{6}=\left[(\varphi-\varphi(t)) e_{13}^{T},(1-\dot{\varphi}(t)) e_{2}^{T}-e_{3}^{T}+\dot{\varphi}(t) e_{8}^{T}\right]^{T}$,

$\Omega_{7}=\left[0, e_{1}^{T}-e_{2}^{T}, 0,-e_{1}^{T}-e_{2}^{T}+2 e_{7}^{T}, 0, e_{1}^{T}-e_{2}^{T}+6 e_{7}^{T}-6 e_{9}^{T}\right]^{T}$,

$\Omega_{8}=\left[e_{7}^{T}, 0, e_{7}^{T}-e_{9}^{T}, 0, e_{7}^{T}-3 e_{9}^{T}+2 e_{11}^{T}, 0\right]^{T}$,

$\Omega_{9}=\left[0, e_{2}^{T}-e_{3}^{T}, 0,-e_{2}^{T}-e_{3}^{T}+2 e_{8}^{T}, 0, e_{2}^{T}-e_{3}^{T}+6 e_{8}^{T}-6 e_{10}^{T}\right]^{T}$,

$\Omega_{10}=\left[e_{8}^{T}, 0, e_{8}^{T}-e_{10}^{T}, 0, e_{8}^{T}-3 e_{10}^{T}+2 e_{12}^{T}, 0\right]^{T}$,

$\Omega_{11}=\left[e_{1}^{T}, e_{13}^{T}\right]^{T}, \Omega_{12}=\left[e_{2}^{T}, e_{14}^{T}\right]^{T}, \Omega_{13}=\left[e_{3}^{T}, e_{15}^{T}\right]^{T}$,

$w_{1}=\left[\left(e_{1}-e_{2}\right)^{T},\left(e_{1}+e_{2}-2 e_{7}\right)^{T},\left(e_{1}-e_{2}+6 e_{7}-\right.\right.$ $\left.\left.6 e_{9}\right)^{T},\left(e_{1}+e_{2}-12 e_{7}+30 e_{9}-20 e_{11}\right)^{T}\right]^{T}$,

$w_{2}=\left[\left(e_{2}-e_{3}\right)^{T},\left(e_{2}+e_{3}-2 e_{8}\right)^{T},\left(e_{2}-e_{3}+6 e_{8}-\right.\right.$

$\left.\left.6 e_{10}\right)^{T},\left(e_{2}+e_{3}-12 e_{8}+30 e_{10}-20 e_{12}\right)^{T}\right]^{T}, Q_{31}=Q_{3 p}$,

$Q_{41}=Q_{4 p}, Q_{32}=Q_{3 p}-\varphi Q_{3 q}, Q_{42}=Q_{4 p}+\varphi Q_{4 q}$,

$N_{1,1}=\left.N_{1}\right|_{\dot{\varphi}(t)=\mu_{1}}, N_{1,2}=\left.N_{1}\right|_{\dot{\varphi}(t)=\mu_{2}}$,

$N_{2,1}=\left.N_{2}\right|_{\dot{\varphi}(t)=\mu_{1}}, N_{2,2}=\left.N_{2}\right|_{\dot{\varphi}(t)=\mu_{2}}$,

$N_{1}=M_{1}+\dot{\varphi}(t) Q_{3 q}+\left[\begin{array}{cc}0 & Z_{1} \\ Z_{1} & 0\end{array}\right]$,

$N_{2}=M_{1}+\dot{\varphi}(t) Q_{4 q}+\left[\begin{array}{cc}0 & Z_{2} \\ Z_{2} & 0\end{array}\right]$,

$\hat{Q}_{3 q}=\operatorname{diag}\left\{N_{1}, 3 N_{1}, 5 N_{1}\right\}, \hat{Q}_{4 q}=\operatorname{diag}\left\{N_{2}, 3 N_{2}, 5 N_{2}\right\}$,

$\Xi_{\alpha_{1}}=\left[\begin{array}{cc}-R_{\alpha_{1}} \bar{E}_{1} & R_{\alpha_{1}} \vec{E}_{2} \\ * & -R_{\alpha_{1}}\end{array}\right] \quad\left(\alpha_{1}=1,2,3\right)$,

$\bar{E}_{1}=I_{N} \otimes E_{1}, \bar{E}_{2}=I_{N} \otimes E_{2}, E_{1}=\operatorname{diag}\left\{e_{1}^{+} e_{1}^{-}, \cdots, e_{n}^{+} e_{n}^{-}\right\}$,

$E_{2}=\operatorname{diag}\left\{\frac{e_{1}^{+}+e_{1}^{-}}{2}, \cdots, \frac{e_{n}^{+}+e_{n}^{-}}{2}\right\}$.

If $i \in S_{\mathrm{k}}^{i}$,

$$
P_{j}-P_{i}-V_{i j} \leq 0(\forall j \in \mathcal{S}, j \neq i),
$$




$$
\begin{gathered}
{\left[\begin{array}{cc}
\hat{\Phi}\left(\mu_{1}, 0\right) & w_{1}^{T} Y_{2} \\
* & -\widehat{M}_{2}
\end{array}\right]<0,} \\
{\left[\begin{array}{cc}
\hat{\Phi}\left(\mu_{1}, \varphi\right) & w_{2}^{T} Y_{1}^{T} \\
* & -\widehat{M}_{2}
\end{array}\right]<0,} \\
{\left[\begin{array}{cc}
\hat{\Phi}\left(\mu_{2}, 0\right) & w_{1}^{T} Y_{2} \\
* & -\widehat{M}_{2}
\end{array}\right]<0,} \\
{\left[\begin{array}{cc}
\hat{\Phi}\left(\mu_{2}, \varphi\right) & w_{2}^{T} Y_{1}^{T} \\
* & -\widehat{M}_{2}
\end{array}\right]<0,} \\
{\left[\begin{array}{cc}
U_{2} & X_{1} \\
* & U_{3}
\end{array}\right] \geq 0,\left[\begin{array}{cc}
\hat{Q}_{3 q} & X_{2} \\
* & \hat{Q}_{4 q}
\end{array}\right] \geq 0,}
\end{gathered}
$$

$\zeta_{0}>0, \zeta_{1}>0, Q_{k, l}>0, N_{\rho, l}>0(k=3,4, \rho, l=1,2)$,

where

$\hat{\Phi}\left(\mu_{1}, 0\right)=\left.\Xi(\dot{\varphi}(t), \varphi(t))\right|_{\dot{\varphi}(t)=\mu_{1}, \varphi(t)=0}+\Xi_{t}+\hat{\Xi}_{i i}$, $\hat{\Phi}\left(\mu_{1}, \varphi\right)=\left.\Xi(\dot{\varphi}(t), \varphi(t))\right|_{\dot{\varphi}(t)=\mu_{1}, \varphi(t)=\varphi}+\Xi_{t}+\hat{\Xi}_{i i}$, $\hat{\Phi}\left(\mu_{2}, 0\right)=\left.\Xi(\dot{\varphi}(t), \varphi(t))\right|_{\dot{\varphi}(t)=\mu_{2}, \varphi(t)=0}+\Xi_{t}+\hat{\Xi}_{i i}$, $\hat{\Phi}\left(\mu_{2}, \varphi\right)=\left.\Xi(\dot{\varphi}(t), \varphi(t))\right|_{\dot{\varphi}(t)=\mu_{2}, \varphi(t)=\varphi}+\Xi_{t}+\hat{\Xi}_{i i}$,

In matrices $\hat{\Phi}\left(\mu_{1}, 0\right), \hat{\Phi}\left(\mu_{2}, 0\right), \hat{\Phi}\left(\mu_{1}, \varphi\right)$ and $\hat{\Phi}\left(\mu_{2}, \varphi\right)$, only the element $\hat{\Xi}_{i i}$ is different from $\Xi_{i i}$, and the other elements are the same as the elements in $\Phi\left(\mu_{1}, 0\right), \Phi\left(\mu_{2}, 0\right), \Phi\left(\mu_{1}, \varphi\right)$ and $\Phi\left(\mu_{2}, \varphi\right)$.

$\hat{\Xi}_{i i}=e_{1}^{T}\left[\sum_{j \in S_{k}^{i}, j \neq i}^{\mathcal{M}}\left[\underline{\pi}_{i j}\left(P_{j}-P_{i}\right)+2 \varpi_{i j} V_{i j}\right]\right.$ $\left.+\underline{\pi}_{i}\left(P_{\varepsilon}-P_{i}\right)+2 \hat{\Theta}_{i} V_{i \varepsilon}\right] e_{1}, \forall \varepsilon \in S_{\mathrm{uk}}^{i}$.

Moreover, the feedback gain matrices are given as follows

$$
K_{i}=F^{-1} J_{i}(i \in \mathcal{S}) .
$$

Proof Choose the DPTLKF for NMASs with generally uncertain switching topologies as follows $V(t, i)=\sum_{\hat{r}=1}^{8} V_{\hat{r}}(t, i)$

$$
\begin{aligned}
V_{1}(t, i)= & v^{T}(t) P_{i} v(t) \\
V_{2}(t, i)= & \int_{t-\varphi(t)}^{t} \eta^{T}(s) Q_{1} \eta(s) d s+\int_{t-\varphi}^{t} \eta^{T}(s) Q_{2} \eta(s) d s \\
V_{3}(t, i)= & \int_{t-\varphi}^{t} v^{T}(s) d s R \int_{t-\varphi}^{t} v(s) d s \\
V_{4}(t, i)= & w_{1}^{T}(t) U_{1} w_{1}(t)+\varphi(t) w_{2}^{T}(t) U_{2} w_{2}(t) \\
& +(\varphi-\varphi(t)) w_{3}^{T}(t) U_{3} w_{3}(t) \\
V_{5}(t, i)= & \int_{t-\varphi(t)}^{t} w_{4}^{T}(s) Q_{3}(\varphi(t)) w_{4}(s) d s \\
& +\int_{t-\varphi}^{t-\varphi(t)} w_{4}^{T}(s) Q_{4}(\varphi(t)) w_{4}(s) d s \\
V_{6}(t, i)= & \int_{-\varphi}^{0} \int_{t+\theta}^{t} w_{4}^{T}(s) M_{1} w_{4}(s) d s d \theta \\
V_{7}(t, i)= & \varphi \int_{-\varphi}^{0} \int_{t+\theta}^{t} \dot{v}^{T}(s) M_{2} \dot{v}(s) d s d \theta
\end{aligned}
$$

$$
\begin{aligned}
V_{8}(t, i)= & h^{2} \int_{q h}^{t} \dot{v}^{T}(s) M_{3} \dot{v}(s) d s \\
& -\frac{\pi^{2}}{4} \int_{q h}^{t}(v(s)-v(q h))^{T} M_{3}(v(s)-v(q h)) d s,
\end{aligned}
$$

where

$\eta(t)=\left[v^{T}(t), g^{T}(v(t))\right]^{T}, \quad w_{1}(t)=\left[v^{T}(t), v^{T}(t-\right.$ $\left.\varphi(t)), \int_{t-\varphi(t)}^{t} v^{T}(s) d s, \int_{t-\varphi}^{t-\varphi(t)} v^{T}(s) d s\right]^{T}$,

$w_{2}(t)=\left[v^{T}(t), \frac{1}{\varphi(t)} \int_{t-\varphi(t)}^{t} v^{T}(s) d s\right]^{T}, \quad w_{3}(t)=$ $\left[v^{T}(t), \frac{1}{\varphi-\varphi(t)} \int_{t-\varphi}^{t-\varphi(t)} v^{T}(s) d s\right]^{T}, w_{4}(t)=\left[v^{T}(t), \dot{v}^{T}(t)\right]^{T}$, $Q_{3}(\varphi(t))=Q_{3 p}-\varphi(t) Q_{3 q}, Q_{4}(\varphi(t))=Q_{4 p}+(\varphi-\varphi(t)) Q_{4 q}$.

In order to prove the positive definiteness of $\operatorname{LKF} V(t, i)$, $V_{4}(t, i)$ is rewritten as the following form

$$
\begin{aligned}
V_{4}(t, i)= & w_{1}^{T}(t)\left[U_{1}+\varphi(t)\left(\Lambda_{1}+\frac{1}{\varphi(t)} \Lambda_{2}\right)^{T} U_{2}\left(\Lambda_{1}+\frac{1}{\varphi(t)} \Lambda_{2}\right)\right. \\
& +(\varphi-\varphi(t))\left(\Lambda_{1}+\frac{1}{\varphi-\varphi(t)} \Lambda_{3}\right)^{T} U_{3}\left(\Lambda_{1}\right. \\
& \left.\left.+\frac{1}{\varphi-\varphi(t)} \Lambda_{3}\right)\right] w_{1}(t) \\
= & w_{1}^{T}(t)\left[U_{1}+\varphi \Lambda_{1}^{T}\left(\delta U_{2}+(1-\delta) U_{3}\right) \Lambda_{1}\right. \\
& +\operatorname{Sym}\left\{\Lambda_{1}^{T} U_{2} \Lambda_{2}+\Lambda_{1}^{T} U_{3} \Lambda_{3}\right\} \\
& \left.+\frac{1}{\varphi}\left(\frac{1}{\delta} \Lambda_{2}^{T} U_{2} \Lambda_{2}+\frac{1}{1-\delta} \Lambda_{3}^{T} U_{3} \Lambda_{3}\right)\right] w_{1}(t)
\end{aligned}
$$

where

$\Lambda_{1}=\left[\begin{array}{ll}\tilde{e}_{1}^{T} & 0\end{array}\right]^{T}, \Lambda_{2}=\left[\begin{array}{ll}0 & \tilde{e}_{3}^{T}\end{array}\right]^{T}, \Lambda_{3}=\left[\begin{array}{ll}0 & \tilde{e}_{4}^{T}\end{array}\right]^{T}$,

$\tilde{e}_{\alpha}=\left[\begin{array}{lll}0_{N n \times(\alpha-1) N n} I_{N n \times N n} & 0_{N n \times(4-\alpha) N n}\end{array}\right], \alpha=1,2,3,4$.

For $U_{2}>0, U_{3}>0$, any matrix $X_{1}$ and $\left[\begin{array}{cc}U_{2} & X_{1} \\ * & U_{3}\end{array}\right] \geq$ 0 , one obtains

$\frac{1}{\delta} \Lambda_{2}^{T} U_{2} \Lambda_{2}+\frac{1}{1-\delta} \Lambda_{3}^{T} U_{3} \Lambda_{3} \geq\left[\begin{array}{c}\Lambda_{2} \\ \Lambda_{3}\end{array}\right]^{T}\left[\begin{array}{cc}U_{2} & X_{1} \\ * & U_{3}\end{array}\right]\left[\begin{array}{c}\Lambda_{2} \\ \Lambda_{3}\end{array}\right]$.

Thus we have

$$
V_{4}(t, i) \geq w_{1}^{T}(t) \zeta_{\delta} w_{1}(t)
$$

where

$$
\begin{aligned}
& \zeta_{\delta}=U_{1}+\varphi \Lambda_{1}^{T}\left(\delta U_{2}+(1-\delta) U_{3}\right) \Lambda_{1}+\operatorname{Sym}\left\{\Lambda_{1}^{T} U_{2} \Lambda_{2}\right. \\
& \left.+\Lambda_{1}^{T} U_{3} \Lambda_{3}\right\}+\frac{1}{\varphi}\left[\begin{array}{c}
\Lambda_{2} \\
\Lambda_{3}
\end{array}\right]^{T}\left[\begin{array}{cc}
U_{2} & X_{1} \\
* & U_{3}
\end{array}\right]\left[\begin{array}{l}
\Lambda_{2} \\
\Lambda_{3}
\end{array}\right]
\end{aligned}
$$

From $\zeta_{0}>0$ and $\zeta_{1}>0$, one obtains $V_{4}(t, i)>0$. From $Q_{k, l}>0(k=3,4, l=1,2)$, one obtains $V_{5}(t, i)>0$. From Lemma 1 , one obtains $V_{8}(t, i) \geq 0 . \mathcal{L}$ is defined as the weak infinitesimal operator. Then, calculating the time derivative of $V(t, i)$, one has

$$
\begin{aligned}
& \mathcal{L} V_{1}(t, i)=2 v^{T}(t) P_{i} \dot{v}(t)+\sum_{j=1}^{\mathcal{M}} \pi_{i j} v^{T}(t) P_{j} v(t), \\
& \mathcal{L} V_{2}(t, i)=\eta^{T}(t) Q_{1} \eta(t)-(1-\dot{\varphi}(t)) \eta^{T}(t-\varphi(t)) Q_{1} \\
& \eta(t-\varphi(t))+\eta^{T}(t) Q_{2} \eta(t)-\eta^{T}(t-\varphi) Q_{2} \eta(t-\varphi), \\
& \mathcal{L} V_{3}(t, i)=2\left[\varphi(t) \times \frac{1}{\varphi(t)} \int_{t-\varphi(t)}^{t} v^{T}(s) d s+(\varphi-\varphi(t))\right.
\end{aligned}
$$




$$
\begin{aligned}
& \left.\times \frac{1}{\varphi-\varphi(t)} \int_{t-\varphi}^{t-\varphi(t)} v^{T}(s) d s\right] R(v(t)-v(t-\varphi)), \\
& \mathcal{L} V_{4}(t, i)=2 w_{1}^{T}(t) U_{1} \dot{w}_{1}(t)+\dot{\varphi}(t) w_{2}^{T}(t) U_{2} w_{2}(t) \\
& +2 \varphi(t) w_{2}^{T}(t) U_{2} \dot{w}_{2}(t)-\dot{\varphi}(t) w_{3}^{T}(t) U_{3} w_{3}(t)+2(\varphi \\
& -\varphi(t)) w_{3}^{T}(t) U_{3} \dot{w}_{3}(t),
\end{aligned}
$$

$\mathcal{L} V_{5}(t, i)=w_{4}^{T}(t)\left(Q_{3 p}-\varphi(t) Q_{3 q}\right) w_{4}(t)-(1-\dot{\varphi}(t)) w_{4}^{T}(t$

$-\varphi(t))\left(Q_{3 p}-\varphi(t) Q_{3 q}\right) w_{4}(t-\varphi(t))+(1-\dot{\varphi}(t)) w_{4}^{T}(t$

$-\varphi(t))\left(Q_{4 p}+(\varphi-\varphi(t)) Q_{4 q}\right) w_{4}(t-\varphi(t))-w_{4}^{T}(t$

$-\varphi)\left(Q_{4 p}+(\varphi-\varphi(t)) Q_{4 q}\right) w_{4}(t-\varphi)-\dot{\varphi}(t) \int_{t-\varphi(t)}^{t} w_{4}^{T}(s)$

$\times Q_{3 q} w_{4}(s) d s-\dot{\varphi}(t) \int_{t-\varphi}^{t-\varphi(t)} w_{4}^{T}(s) Q_{4 q} w_{4}(s) d s$,

$\mathcal{L} V_{6}(t, i)=\varphi w_{4}^{T}(t) M_{1} w_{4}(t)-\int_{t-\varphi}^{t} w_{4}^{T}(s) M_{1} w_{4}(s) d s$,

$\mathcal{L} V_{7}(t, i)=\varphi^{2} \dot{v}^{T}(t) M_{2} \dot{v}(t)-\varphi \int_{t-\varphi}^{t} \dot{v}^{T}(s) M_{2} \dot{v}(s) d s$,

$\mathcal{L} V_{8}(t, i)$

$=h^{2} \dot{v}^{T}(t) M_{3} \dot{v}(t)-\frac{\pi^{2}}{4}(v(t)-v(q h))^{T} M_{3}(v(t)-v(q h))$.

By using Lemma 2, one obtains

$-\varphi \int_{t-\varphi}^{t} \dot{v}^{T}(s) M_{2} \dot{v}(s) d s$

$\leq-\xi^{T}(t)\left\{\left[\begin{array}{l}w_{1} \\ w_{2}\end{array}\right]^{T} \hat{\phi}_{1}(\delta, 1-\delta)\left[\begin{array}{l}w_{1} \\ w_{2}\end{array}\right]\right\} \xi(t)$

$\leq-\xi^{T}(t)\left\{\left[\begin{array}{l}w_{1} \\ w_{2}\end{array}\right]^{T} \hat{\phi}_{2}(\delta, 1-\delta)\left[\begin{array}{l}w_{1} \\ w_{2}\end{array}\right]\right\} \xi(t)$

$+\xi^{T}(t)\left[\delta w_{2}^{T} Y_{1}^{T} \widehat{M}_{2}^{-1} Y_{1} w_{2}+(1-\delta) w_{1}^{T} Y_{2} \widehat{M}_{2}^{-1} Y_{2}^{T} w_{1}\right] \xi(t)$,

where

$\hat{\Omega}_{1}=\widehat{M}_{2}-Y_{2} \widehat{M}_{2}^{-1} Y_{2}^{T}, \hat{\Omega}_{2}=\widehat{M}_{2}-Y_{1}^{T} \widehat{M}_{2}^{-1} Y_{1}$,

$\hat{\phi}_{1}(\delta, 1-\delta)=\left[\begin{array}{cc}\widehat{M}_{2}+(1-\delta) \hat{\Omega}_{1} & (1-\delta) Y_{1}+\delta Y_{2} \\ * & \widehat{M}_{2}+\delta \hat{\Omega}_{2}\end{array}\right]$,

$\widehat{M}_{2}, \hat{\phi}_{2}(\delta, 1-\delta), \delta, w_{1}, w_{2}$ are given in Theorem 1 .

By using the integral formula, the following zero equalities with $Z_{l}(l=1,2)$ hold

$$
\begin{aligned}
& 0=v^{T}(t) Z_{1} v(t)-v^{T}(t-\varphi(t)) Z_{1} v(t-\varphi(t)) \\
& -2 \int_{t-\varphi(t)}^{t} v^{T}(s) Z_{1} \dot{v}(s) d s \\
& 0=v^{T}(t-\varphi(t)) Z_{2} v(t-\varphi(t))-v^{T}(t-\varphi) Z_{2} v(t-\varphi) \\
& -2 \int_{t-\varphi}^{t-\varphi(t)} v^{T}(s) Z_{2} \dot{v}(s) d s .
\end{aligned}
$$

From (46), one obtains

$$
-\int_{t-\varphi}^{t} w_{4}^{T}(s) M_{1} w_{4}(s) d s
$$

$=-\int_{t-\varphi}^{t-\varphi(t)} w_{4}^{T}(s) M_{1} w_{4}(s) d s-\int_{t-\varphi(t)}^{t} w_{4}^{T}(s) M_{1} w_{4}(s) d s$.

Considering the single integral terms in (45), (46) and (52), one has

$\Theta_{1}$

$=-\int_{t-\varphi(t)}^{t} w_{4}^{T}(s)\left(M_{1}+\dot{\varphi}(t) Q_{3 q}+\left[\begin{array}{cc}0 & Z_{1} \\ Z_{1} & 0\end{array}\right]\right) w_{4}(s) d s$,

$\Theta_{2}$

$=-\int_{t-\varphi}^{t-\varphi(t)} w_{4}^{T}(s)\left(M_{1}+\dot{\varphi}(t) Q_{4 q}+\left[\begin{array}{cc}0 & Z_{2} \\ Z_{2} & 0\end{array}\right]\right) w_{4}(s) d s$.

From integral inequality in Remark 4 of [60], reciprocal convexity in [61], the upper bound of the (53) and (54) is obtained as follows

$$
\begin{aligned}
& \Theta_{1}+\Theta_{2} \\
& \leq-\xi^{T}(t)\left[\frac{1}{\varphi(t)}\left(\Omega_{7}+\varphi(t) \Omega_{8}\right)^{T} \hat{Q}_{3 q}\left(\Omega_{7}+\varphi(t) \Omega_{8}\right)\right. \\
& +\frac{1}{\varphi-\varphi(t)}\left(\Omega_{9}+(\varphi-\varphi(t)) \Omega_{10}\right)^{T} \hat{Q}_{4 q}\left(\Omega_{9}\right. \\
& \left.\left.+(\varphi-\varphi(t)) \Omega_{10}\right)\right] \xi(t) \\
& \leq-\xi^{T}(t)\left[\operatorname{Sym}\left\{\Omega_{7}^{T} \hat{Q}_{3 q} \Omega_{8}+\Omega_{9}^{T} \hat{Q}_{4 q} \Omega_{10}\right\}+\delta \varphi \Omega_{8}^{T} \hat{Q}_{3 q} \Omega_{8}\right. \\
& +(1-\delta) \varphi \Omega_{10}^{T} \hat{Q}_{4 q} \Omega_{10}+\frac{1}{\varphi}\left[\begin{array}{c}
\Omega_{7} \\
\Omega_{9}
\end{array}\right]^{T}\left[\begin{array}{cc}
\hat{Q}_{3 q} & X_{2} \\
* & \hat{Q}_{4 q}
\end{array}\right] \\
& \left.\times\left[\begin{array}{c}
\Omega_{7} \\
\Omega_{9}
\end{array}\right]\right] \xi(t) .
\end{aligned}
$$

From Assumption 3, one obtains

$$
\begin{aligned}
& \eta^{T}(t)\left[\begin{array}{cc}
-R_{1} \bar{E}_{1} & R_{1} \bar{E}_{2} \\
* & -R_{1}
\end{array}\right] \eta(t) \geq 0, \\
& \eta^{T}(t-\varphi(t))\left[\begin{array}{cc}
-R_{2} \bar{E}_{1} & R_{2} \bar{E}_{2} \\
* & -R_{2}
\end{array}\right] \eta(t-\varphi(t)) \geq 0, \\
& \eta^{T}(t-\varphi)\left[\begin{array}{cc}
-R_{3} \bar{E}_{1} & R_{3} \bar{E}_{2} \\
* & -R_{3}
\end{array}\right] \eta(t-\varphi) \geq 0 .
\end{aligned}
$$

For invertible matrix $F$ and (9), one obtains

$$
\begin{aligned}
& 2\left[v^{T}(t)\left(I_{N} \otimes F\right)+m \dot{v}^{T}(t)\left(I_{N} \otimes F\right)\right]\left[-\dot{v}(t)+\left(I_{N} \otimes A\right) v(t)\right. \\
& +\left(I_{N} \otimes B\right) g(v(t))+\left(I_{N} \otimes C\right) g(v(t-\varphi(t))) \\
& \left.-\left(G_{i} \otimes K_{i}\right) v(t-d(t))-\left(G_{i} \otimes K_{i}\right) y(t-d(t))\right]=0 . \quad(59)
\end{aligned}
$$

Assuming $J_{i}=F K_{i}$, one obtains

$$
\begin{aligned}
& 2\left[v^{T}(t)+m \dot{v}^{T}(t)\right]\left[-\left(I_{N} \otimes F\right) \dot{v}(t)+\left(I_{N} \otimes F A\right) v(t)\right. \\
& +\left(I_{N} \otimes F B\right) g(v(t))+\left(I_{N} \otimes F C\right) g(v(t-\varphi(t))) \\
& \left.-\left(G_{i} \otimes J_{i}\right) v(t-d(t))-\left(G_{i} \otimes J_{i}\right) y(t-d(t))\right]=0 .
\end{aligned}
$$

From (4), we obtain the following formula

$$
\sum_{k=1}^{N} y_{k}^{T}(q h) \Omega y_{k}(q h)=y^{T}(q h)\left(I_{N} \otimes \Omega\right) y(q h)
$$




$$
\leq \omega^{T}(q h)(\Theta \otimes \Omega) \omega(q h)=\sum_{k=1}^{N} \theta_{k} \omega_{k}^{T}(q h) \Omega \omega_{k}(q h),
$$

where $\omega(q h)=\left(G_{i} \otimes I_{n}\right) v(q h)+\left(G_{i} \otimes I_{n}\right) y(q h)$, $y(q h)=\left[y_{1}^{T}(q h), y_{2}^{T}(q h), \cdots, y_{N}^{T}(q h)\right]^{T}, \omega(q h)=$ $\left[\omega_{1}^{T}(q h), \omega_{2}^{T}(q h), \cdots, \omega_{N}^{T}(q h)\right]^{T}$. We have

$$
\begin{aligned}
& y^{T}(t-d(t))\left(I_{N} \otimes \Omega\right) y(t-d(t)) \leq\left[v^{T}(t-d(t))\right. \\
& \left.+y^{T}(t-d(t))\right]\left(G_{i}^{T} \Theta G_{i} \otimes \Omega\right)[v(t-d(t))+y(t-d(t))]
\end{aligned}
$$

Then combining (29) to (62), one obtains $\mathcal{E}\{\mathcal{L} V(t, i)\} \leq$ $\mathcal{E}\left\{\xi^{T}(t) \wp(t) \xi(t)\right\}<0$,

where

$$
\begin{aligned}
\wp(s) & =\bar{\phi}(\dot{\varphi}(t), \varphi(t))+\delta w_{2}^{T} Y_{1}^{T} \widehat{M}_{2}^{-1} Y_{1} w_{2} \\
& +(1-\delta) w_{1}^{T} Y_{2} \widehat{M}_{2}^{-1} Y_{2}^{T} w_{1},
\end{aligned}
$$

$\bar{\phi}(\dot{\varphi}(t), \varphi(t))=\Xi_{t}+\Xi(\dot{\varphi}(t), \varphi(t))+e_{1}^{T} \sum_{j=1}^{\mathcal{M}} \pi_{i j} P_{j} e_{1}$, $\xi(t)=\left[v^{T}(t), v^{T}(t-\varphi(t)), v^{T}(t-\varphi), g^{T}(v(t))\right.$, $g^{T}(v(t-\varphi(t))), g^{T}(v(t-\varphi)), \frac{1}{\varphi(t)} \int_{t-\varphi(t)}^{t} v^{T}(s) d s$, $\frac{1}{\varphi-\varphi(t)} \int_{t-\varphi}^{t-\varphi(t)} v^{T}(s) d s, \frac{2}{(\varphi(t))^{2}} \int_{t-\varphi(t)}^{t} \int_{\theta}^{t} v^{T}(s) d s d \theta$, $\frac{2}{(\varphi-\varphi(t))^{2}} \int_{t-\varphi}^{t-\varphi(t)} \int_{\theta}^{t-\varphi(t)} v^{T}(s) d s d \theta$, $\frac{6}{(\varphi(t))^{3}} \int_{t-\varphi(t)}^{t} \int_{\theta}^{t} \int_{\alpha}^{t} v^{T}(s) d s d \alpha d \theta$, $\frac{6}{(\varphi-\varphi(t))^{3}} \int_{t-\varphi}^{t-\varphi(t)} \int_{\theta}^{t-\varphi(t)} \int_{\alpha}^{t-\varphi(t)} v^{T}(s) d s d \alpha d \theta, \dot{v}^{T}(t)$, $\left.\dot{v}^{T}(t-\varphi(t)), \dot{v}^{T}(t-\varphi), v^{T}(t-d(t)), y^{T}(t-d(t))\right]^{T}$.

According to convex theory, $\mathcal{E}\left\{\xi^{T}(t) \wp(t) \xi(t)\right\}<0$ could be satisfied for all $(\dot{\varphi}(t)), \varphi(t)) \in\left[\mu_{1}, \mu_{2}\right] \times[0, \varphi]$ if it is satisfied at the vertices of the interval $\left[\mu_{1}, \mu_{2}\right] \times[0, \varphi]$.

According to Schur complement, if $\mathcal{E}\left\{\bar{\phi}^{\gamma}\right\}<0(\gamma=$ $1,2,3,4)$ hold, then $\mathcal{E}\left\{\xi^{T}(t) \wp(t) \xi(t)\right\}<0$ holds, where

$$
\begin{aligned}
\bar{\phi}^{1} & =\left[\begin{array}{cc}
\bar{\phi}\left(\mu_{1}, 0\right) & w_{1}^{T} Y_{2} \\
* & -\widehat{M}_{2}
\end{array}\right], \bar{\phi}^{2}=\left[\begin{array}{cc}
\bar{\phi}\left(\mu_{1}, \varphi\right) & w_{2}^{T} Y_{1}^{T} \\
* & -\widehat{M}_{2}
\end{array}\right], \\
\bar{\phi}^{3} & =\left[\begin{array}{cc}
\bar{\phi}\left(\mu_{2}, 0\right) & w_{1}^{T} Y_{2} \\
* & -\widehat{M}_{2}
\end{array}\right], \bar{\phi}^{4}=\left[\begin{array}{cc}
\bar{\phi}\left(\mu_{2}, \varphi\right) & w_{2}^{T} Y_{1}^{T} \\
* & -\widehat{M}_{2}
\end{array}\right] .
\end{aligned}
$$

$\bar{\phi}\left(\mu_{1}, 0\right)=\left.\bar{\phi}(\dot{\varphi}(t), \varphi(t))\right|_{\dot{\varphi}(t)=\mu_{1}, \varphi(t)=0}$, $\bar{\phi}\left(\mu_{1}, \varphi\right)=\left.\bar{\phi}(\dot{\varphi}(t), \varphi(t))\right|_{\dot{\varphi}(t)=\mu_{1}, \varphi(t)=\varphi}$, $\bar{\phi}\left(\mu_{2}, 0\right)=\left.\bar{\phi}(\dot{\varphi}(t), \varphi(t))\right|_{\dot{\varphi}(t)=\mu_{2}, \varphi(t)=0}$, $\bar{\phi}\left(\mu_{2}, \varphi\right)=\left.\bar{\phi}(\dot{\varphi}(t), \varphi(t))\right|_{\dot{\varphi}(t)=\mu_{2}, \varphi(t)=\varphi}$.

Thus, we have

$$
\mathcal{E}\{\mathcal{L} V(t, i)\}<-\epsilon v^{T}(t) v(t), t \in[q h,(q+1) h)
$$

for a small enough $\epsilon>0$. For $t_{q}=q h, t_{q+1}=(q+1) h$, and Dynkins formula, we obtain

$$
\begin{aligned}
& \mathcal{E}\left\{V\left(v\left(t_{q+1}^{-}\right), t_{q+1}^{-}, \dot{v}\left(t_{q+1}^{-}\right)\right)\right\}-\mathcal{E}\left\{V\left(v\left(t_{q}\right), t_{q}, \dot{v}\left(t_{q}\right)\right)\right\} \\
& \leq-\epsilon \mathcal{E}\left\{\int_{t_{q}}^{t_{q+1}^{-}}\|v(s)\|^{2} d s\right\} .
\end{aligned}
$$

Similarly, we obtain

$$
\begin{aligned}
& \mathcal{E}\left\{V\left(v\left(t_{q}^{-}\right), t_{q}^{-}, \dot{v}\left(t_{q}^{-}\right)\right)\right\}-\mathcal{E}\left\{V\left(v\left(t_{q-1}\right), t_{q-1}, \dot{v}\left(t_{q-1}\right)\right)\right\} \\
& \leq-\epsilon \mathcal{E}\left\{\int_{t_{q-1}}^{t_{q}^{-}}\|v(s)\|^{2} d s\right\}
\end{aligned}
$$

$$
\begin{aligned}
& \cdots \\
& \mathcal{E}\left\{V\left(v\left(t_{1}^{-}\right), t_{1}^{-}, \dot{v}\left(t_{1}^{-}\right)\right)\right\}-\mathcal{E}\left\{V\left(v_{0}, t_{0}, \dot{v}_{0}\right)\right\} \\
& \leq-\epsilon \mathcal{E}\left\{\int_{0}^{t_{1}^{-}}\|v(s)\|^{2} d s\right\} .
\end{aligned}
$$

Therefore, one obtains

$$
\sum_{q=0}^{\infty} \mathcal{E}\left\{\int_{t_{q}}^{t_{q+1}^{-}}\|v(s)\|^{2} d s\right\} \leq \epsilon^{-1} \mathcal{E}\left\{V\left(v_{0}, 0, \dot{v}(0)\right)\right\},
$$

which means that $\lim _{t \rightarrow \infty} \mathcal{E}\|v(t)\|^{2}=0$. Thus, with the protocol (5) and Definition 1 , the systems (9) reach leader-following consensus.

Now, we will prove that $\mathcal{E}\left\{\bar{\phi}^{\gamma}\right\}<0(\gamma=1,2,3,4)$ hold if (13)-(20) are satisfied.

Assume $\hat{\Pi}_{1}^{\gamma}=e_{1}\left(\Xi_{t}+\Xi^{\gamma}(\dot{\varphi}(t), \varphi(t))\right) e_{1}^{T}, \hat{\Omega}_{1}^{\gamma}=\hat{\Pi}_{1}^{\gamma}+$ $\sum_{j=1}^{\mathcal{M}} \pi_{i j} P_{j}(\gamma=1,2,3,4)$,

where $\Xi^{1}(\dot{\varphi}(t), \varphi(t))=\left.\Xi(\dot{\varphi}(t), \varphi(t))\right|_{\dot{\varphi}(t)=\mu_{1}, \varphi(t)=0}$,

$\Xi^{2}(\dot{\varphi}(t), \varphi(t))=\left.\Xi(\dot{\varphi}(t), \varphi(t))\right|_{\dot{\varphi}(t)=\mu_{1}, \varphi(t)=\varphi}$,

$\Xi^{3}(\dot{\varphi}(t), \varphi(t))=\left.\Xi(\dot{\varphi}(t), \varphi(t))\right|_{\dot{\varphi}(t)=\mu_{2}, \varphi(t)=0}$,

$\Xi^{4}(\dot{\varphi}(t), \varphi(t))=\left.\Xi(\dot{\varphi}(t), \varphi(t))\right|_{\dot{\varphi}(t)=\mu_{2}, \varphi(t)=\varphi}$.

If $i \notin S_{\mathrm{k}}^{i}$, one obtains $\sum_{j=1} \pi_{i j} P_{j}=\sum_{j \in S_{\mathrm{k}}^{i}} \pi_{i j} P_{j}+\pi_{i i} P_{i}$

$+\sum_{j \in S_{\mathrm{uk}}^{i}, j \neq i} \pi_{i j} P_{j}$, where $\pi_{i j} \geq 0, j \neq i$ and $0 \leq \sum_{j \in S_{\mathrm{uk}}^{i}, j \neq i} \pi_{i j}$ $=-\pi_{i i}-\sum_{j \in S_{\mathrm{k}}^{i}} \pi_{i j}$.

From $P_{j}-P_{i}-V_{i j} \leq 0\left(\forall j \in S_{\mathrm{k}}^{i}\right)$ and $P_{j}-P_{i} \leq 0(\forall j \in$ $\left.S_{\mathrm{uk}}^{i}, j \neq i\right)$, one has

$$
\begin{aligned}
& \sum_{j \in S_{\mathrm{k}}^{i}} \pi_{i j} P_{j}+\pi_{i i} P_{i}+\sum_{j \in S_{\mathrm{uk}}^{i}, j \neq i} \pi_{i j} P_{j} \\
& \leq \sum_{j \in S_{\mathrm{k}}^{i}} \pi_{i j} P_{j}+\pi_{i i} P_{i}+\sum_{j \in S_{\mathrm{uk}}^{i}, j \neq i} \pi_{i j} P_{i} \\
& =\sum_{j \in S_{\mathrm{k}}^{i}} \pi_{i j} P_{j}+\pi_{i i} P_{i}+\left(-\pi_{i i}-\sum_{j \in S_{\mathrm{k}}^{i}} \pi_{i j}\right) P_{i} \\
& =\sum_{j \in S_{\mathrm{k}}^{i}}\left(\underline{\pi}_{i j}+\varpi_{i j}+\triangle_{i j}\right)\left(P_{j}-P_{i}\right) \\
& =\sum_{j \in S_{\mathrm{k}}^{i}}\left[\underline{\pi}_{i j}\left(P_{j}-P_{i}\right)+\left(\varpi_{i j}+\triangle_{i j}\right)\left(P_{j}-P_{i}\right)\right] .
\end{aligned}
$$

Noting that $V_{i j} \geq 0, \Delta_{i j} \in\left[-\varpi_{i j}, \varpi_{i j}\right], \forall j \in S_{\mathrm{k}}^{i}$, one has

$\left(\varpi_{i j}+\Delta_{i j}\right)\left(P_{j}-P_{i}\right) \leq\left(\varpi_{i j}+\Delta_{i j}\right) V_{i j} \leq 2 \varpi_{i j} V_{i j}, \forall j \in S_{\mathrm{k}}^{i}$.

For $\gamma=1,2,3,4$, one obtains

$$
\hat{\Omega}_{1}^{\gamma} \leq \hat{\Pi}_{1}^{\gamma}+\sum_{j \in S_{\mathrm{k}}^{i}}\left(\underline{\pi}_{i j}\left(P_{j}-P_{i}\right)+2 \varpi_{i j} V_{i j}\right) .
$$

From (13)-(20), one obtains $\mathcal{E}\left\{\bar{\phi}^{\gamma}\right\}<0(\gamma=1,2,3,4)$.

If $i \in S_{\mathrm{k}}^{i}$, according to $\pi_{i i}=-\sum_{j \in \mathcal{S}, j \neq i} \pi_{i j}$, there is $P_{j} \leq$ $P_{\varepsilon}\left(j, \varepsilon \in S_{\mathrm{uk}}^{i}\right)$, and one has

$$
\sum_{j=1}^{\mathcal{M}} \pi_{i j} P_{j}=\sum_{j \in S_{\mathrm{k}}^{i}, j \neq i} \pi_{i j} P_{j}+\sum_{j \in S_{\mathrm{uk}}^{i}} \pi_{i j} P_{j}+\pi_{i i} P_{i},
$$




$$
\begin{aligned}
& =\sum_{j \in S_{\mathrm{k}}^{i}, j \neq i} \pi_{i j}\left(P_{j}-P_{i}\right)+\sum_{j \in S_{\mathrm{uk}}^{i}} \pi_{i j}\left(P_{j}-P_{i}\right) \\
& \leq \sum_{j \in S_{\mathrm{k}}^{i}, j \neq i} \pi_{i j}\left(P_{j}-P_{i}\right)+\sum_{j \in S_{\mathrm{uk}}^{i}} \pi_{i j}\left(P_{\varepsilon}-P_{i}\right),\left(\varepsilon \in S_{\mathrm{uk}}^{i}\right) .
\end{aligned}
$$

From $P_{j}-P_{i}-V_{i j} \leq 0(\forall j \in \mathcal{S}, j \neq i)$, one has

$$
\sum_{j \in S_{\mathrm{k}}^{i}, j \neq i} \pi_{i j}\left(P_{j}-P_{i}\right) \leq \sum_{j \in S_{\mathrm{k}}^{i}, j \neq i}\left[\underline{\pi}_{i j}\left(P_{j}-P_{i}\right)+2 \varpi_{i j} V_{i j}\right],
$$$$
\sum_{j \in S_{\mathrm{uk}}^{i}} \pi_{i j}\left(P_{\varepsilon}-P_{i}\right) \leq \sum_{j \in S_{\mathrm{uk}}^{i}}\left[\underline{\pi}_{i j}\left(P_{\varepsilon}-P_{i}\right)+2 \varpi_{i j} V_{i \varepsilon}\right] .
$$

From $\pi_{i i}=-\sum_{j \in S_{\mathrm{k}}^{i}, j \neq i} \pi_{i j}-\sum_{j \in S_{\mathrm{uk}}^{i}} \pi_{i j}$, one has

$$
\begin{aligned}
& \sum_{j \in S_{\mathrm{uk}}^{i}} \underline{\pi}_{i j}=-\bar{\pi}_{i i}-\sum_{j \in S_{\mathrm{k}}^{i}, j \neq i} \underline{\pi}_{i j}=\underline{\pi}_{i}, \\
& \sum_{j \in S_{\mathrm{uk}}^{i}} \bar{\pi}_{i j}=-\underline{\pi}_{i i}-\sum_{j \in S_{\mathrm{k}}^{i}, j \neq i} \bar{\pi}_{i j} .
\end{aligned}
$$

Then, one obtains

$$
\begin{aligned}
& \sum_{j \in S_{\mathrm{uk}}^{i}} \varpi_{i j}=\frac{1}{2}\left(-\sum_{j \in S_{\mathrm{uk}}^{i}} \underline{\pi}_{i j}+\sum_{j \in S_{\mathrm{uk}}^{i}} \bar{\pi}_{i j}\right) \\
& =\frac{1}{2}\left(\bar{\pi}_{i i}+\sum_{j \in S_{\mathrm{k}}^{i}, j \neq i} \underline{\pi}_{i j}-\underline{\pi}_{i i}-\sum_{j \in S_{\mathrm{k}}^{i}, j \neq i} \bar{\pi}_{i j}\right) \\
& =\varpi_{i i}-\sum_{j \in S_{\mathrm{k}}^{i}, j \neq i} \varpi_{i j}=\hat{\Theta}_{i} .
\end{aligned}
$$

Thus, one obtains

$$
\sum_{j \in S_{\mathrm{uk}}^{i}} \pi_{i j}\left(P_{j}-P_{i}\right) \leq \underline{\pi}_{i}\left(P_{\varepsilon}-P_{i}\right)+2 \hat{\Theta}_{i} V_{i \varepsilon} .
$$

Hence, one obtains

$$
\begin{aligned}
& \hat{\Omega}_{1}^{\gamma} \leq \hat{\Pi}_{1}^{\gamma}+\underline{\pi}_{i}\left(P_{\varepsilon}-P_{i}\right)+2 \hat{\Theta}_{i} V_{i \varepsilon} \\
& +\sum_{j \in S_{\mathrm{k}}^{i}, j \neq i}\left(\underline{\pi}_{i j}\left(P_{j}-P_{i}\right)+2 \varpi_{i j} V_{i j}\right) .
\end{aligned}
$$

Hence, from (21) to (27), one obtains $\mathcal{E}\left\{\bar{\phi}^{\gamma}\right\}<0(\gamma=$ $1,2,3,4)$

From (60), we obtain the feedback gain matrices as follows

$$
K_{i}=F^{-1} J_{i}(i \in \mathcal{S}) .
$$

Remark 5: Compared with [3], [51], [55], the generally uncertain Markovian switching topologies of MASs are investigated in this article, which is more practical because it is hard to precisely estimate the transition rates due to the influence of device performances and the uncertainties of external environment. Compared with the authors' past article with generally uncertain Markovian jumping [49], [62], without adopting Schur complement and matrix inequality Lemma to deal with generally uncertain Markovian, only one set of relaxation variables is utilized in this article, which could reduce the computational complexity of consensus conditions.

\section{B. Leader-Following Dissipativity Consensus Control}

Under the condition $w_{k}(t) \neq 0$, the strictly $(\mathcal{P}, \mathcal{T}, \mathcal{R})-\rho$ dissipative capability is addressed to reach the leader-following consensus of NMASs (2) and (3).

Theorem 2 Under Assumptions 1-3, the given scalars $\rho>0$ and $m$, matrix $\mathcal{T} \in \mathcal{R}^{N n \times N n}$, symmetric matrices $\mathcal{P} \in$ $\mathcal{R}^{N n \times N n}, \mathcal{P} \leq 0$ and $\mathcal{R} \in \mathcal{R}^{N n \times N n}$, the dissipativitybased consensus control of NMASs (2) and (3) is achieved if there are any matrices $X_{1} \in \mathcal{R}^{2 N n \times 2 N n}, X_{2} \in \mathcal{R}^{6 N n \times 6 N n}$, $Y_{l} \in \mathcal{R}^{4 N n \times 4 N n}, J_{i} \in \mathcal{R}^{N n \times N n}$, invertible matrix $F \in$ $\mathcal{R}^{N n \times N n}$, symmetric matrices $U_{1} \in \mathcal{R}^{4 N n \times 4 N n}, U_{\nu} \in$ $\mathcal{R}^{2 n \times 2 n}, U_{\nu}>0(\nu=2,3), Q_{k p}, Q_{k q} \in \mathcal{R}^{2 N n \times 2 N n}(k=$ $3,4), Z_{l} \in \mathcal{R}^{N n \times N n}(l=1,2)$, symmetric and positive definite matrices $P_{i} \in \mathcal{R}^{N n \times N n}, V_{i j} \in \mathcal{R}^{N n \times N n}, R \in$ $\mathcal{R}^{N n \times N n}, M_{\nu} \in \mathcal{R}^{N n \times N n}(\nu=2,3), M_{1} \in \mathcal{R}^{2 N n \times 2 N n}$, $Q_{l} \in \mathcal{R}^{2 N n \times 2 N n}(l=1,2)$, and diagonal matrices $R_{\alpha_{1}}>$ $0\left(\alpha_{1}=1,2,3\right)$, such that for any $i \in \mathcal{S}$, the following LMIs (82)-(96) are satisfied.

If $i \notin S_{\mathrm{k}}^{i}$,

$$
\begin{aligned}
& P_{j}-P_{i}-V_{i j} \leq 0\left(\forall j \in S_{\mathrm{k}}^{i}\right), \\
& P_{j}-P_{i} \leq 0\left(\forall j \in S_{\mathrm{uk}}^{i}, j \neq i\right), \\
& {\left[\begin{array}{cc}
\tilde{\Phi}\left(\mu_{1}, 0\right) & \tilde{w}_{1}^{T} Y_{2} \\
* & -\widehat{M}_{2}
\end{array}\right]<0,} \\
& {\left[\begin{array}{cc}
\tilde{\Phi}\left(\mu_{1}, \varphi\right) & \tilde{w}_{2}^{T} Y_{1}^{T} \\
* & -\widehat{M}_{2}
\end{array}\right]<0,} \\
& {\left[\begin{array}{cc}
\tilde{\Phi}\left(\mu_{2}, 0\right) & \tilde{w}_{1}^{T} Y_{2} \\
* & -\widehat{M}_{2}
\end{array}\right]<0,} \\
& {\left[\begin{array}{cc}
\tilde{\Phi}\left(\mu_{2}, \varphi\right) & \tilde{w}_{2}^{T} Y_{1}^{T} \\
* & -\widehat{M}_{2}
\end{array}\right]<0,} \\
& {\left[\begin{array}{cc}
U_{2} & X_{1} \\
* & U_{3}
\end{array}\right] \geq 0,\left[\begin{array}{cc}
\hat{Q}_{3 q} & X_{2} \\
* & \hat{Q}_{4 q}
\end{array}\right] \geq 0,}
\end{aligned}
$$

$$
\zeta_{0}>0, \zeta_{1}>0, Q_{k, l}>0, N_{\rho, l}>0(k=3,4, \rho, l=1,2) .
$$

where

$\tilde{\Phi}\left(\mu_{1}, 0\right)=\left.\hat{\Xi}(\dot{\varphi}(t), \varphi(t))\right|_{\dot{\varphi}(t)=\mu_{1}, \varphi(t)=0}+\hat{\Xi}_{t}+\tilde{\Xi}_{i i}$, $\tilde{\Phi}\left(\mu_{1}, \varphi\right)=\left.\hat{\Xi}(\dot{\varphi}(t), \varphi(t))\right|_{\dot{\varphi}(t)=\mu_{1}, \varphi(t)=\varphi}+\hat{\Xi}_{t}+\tilde{\Xi}_{i i}$, $\tilde{\Phi}\left(\mu_{2}, 0\right)=\left.\hat{\Xi}(\dot{\varphi}(t), \varphi(t))\right|_{\dot{\varphi}(t)=\mu_{2}, \varphi(t)=0}+\hat{\Xi}_{t}+\tilde{\Xi}_{i i}$, $\tilde{\Phi}\left(\mu_{2}, \varphi\right)=\left.\hat{\Xi}(\dot{\varphi}(t), \varphi(t))\right|_{\dot{\varphi}(t)=\mu_{2}, \varphi(t)=\varphi}+\hat{\Xi}_{t}+\tilde{\Xi}_{i i}$, $\hat{\Xi}(\dot{\varphi}(t), \varphi(t))=\dot{\varphi}(t) \eta_{2}^{T} Q_{1} \eta_{2}+\operatorname{Sym}\left\{\left[\varphi(t) \hat{e}_{7}^{T}+(\varphi-\right.\right.$ $\left.\left.\varphi(t)) \hat{e}_{8}^{T}\right] R\left(\hat{e}_{1}-\hat{e}_{3}\right)\right\}+\dot{\varphi}(t)\left[\Omega_{3}^{T} U_{2} \Omega_{3}-\Omega_{4}^{T} U_{3} \Omega_{4}\right]+$ $\operatorname{Sym}\left\{\Omega_{1}^{T} U_{1} \Omega_{2}\right\}+\operatorname{Sym}\left\{\Omega_{3}^{T} U_{2} \Omega_{5}\right\}+\operatorname{Sym}\left\{\Omega_{4}^{T} U_{3} \Omega_{6}\right\}+$ $\Omega_{11}^{T}\left(Q_{3 p}-\varphi(t) Q_{3 q}\right) \Omega_{11}-(1-\dot{\varphi}(t)) \Omega_{12}^{T}\left(Q_{3 p}-\right.$ $\left.\varphi(t) Q_{3 q}\right) \Omega_{12}+(1-\dot{\varphi}(t)) \Omega_{12}^{T}\left(Q_{4 p}+(\varphi-\varphi(t)) Q_{4 q}\right) \Omega_{12}-$ $\Omega_{13}^{T}\left(Q_{4 p}+(\varphi-\varphi(t)) Q_{4 q}\right) \Omega_{13}-\operatorname{Sym}\left\{\Omega_{7}^{T} \hat{Q}_{3 q} \Omega_{8}+\right.$ $\left.\Omega_{9}^{T} \hat{Q}_{4 q} \Omega_{10}\right\}-\delta \varphi \Omega_{8}^{T} \hat{Q}_{3 q} \Omega_{8}-(1-\delta) \varphi \Omega_{10}^{T} \hat{Q}_{4 q} \Omega_{10}-$ $\frac{1}{\varphi}\left[\begin{array}{l}\Omega_{7} \\ \Omega_{9}\end{array}\right]^{T}\left[\begin{array}{cc}\hat{Q}_{3 q} & X_{2} \\ * & \hat{Q}_{4 q}\end{array}\right]\left[\begin{array}{l}\Omega_{7} \\ \Omega_{9}\end{array}\right]-\left[\begin{array}{c}\tilde{w}_{1} \\ \tilde{w}_{2}\end{array}\right]^{T} \hat{\phi}_{2}(\delta, 1-$ $\delta)\left[\begin{array}{l}\tilde{w}_{1} \\ \tilde{w}_{2}\end{array}\right], \quad \hat{\Xi}_{t}=\operatorname{Sym}\left\{\hat{e}_{1}^{T} P_{i} \hat{e}_{13}\right\}+\eta_{1}^{T} Q_{1} \eta_{1}-\eta_{2}^{T} Q_{1} \eta_{2}+$ $\eta_{1}^{T} Q_{2} \eta_{1}-\eta_{3}^{T} Q_{2} \eta_{3}+\varphi \Omega_{11}^{T} M_{1} \Omega_{11}+\varphi^{2} \hat{e}_{13}^{T} M_{2} \hat{e}_{13}+$ $h^{2} \hat{e}_{13}^{T} M_{3} \hat{e}_{13}-\frac{\pi^{2}}{4}\left(\hat{e}_{1}-\hat{e}_{16}\right)^{T} M_{3}\left(\hat{e}_{1}-\hat{e}_{16}\right)+\hat{e}_{1}^{T} Z_{1} \hat{e}_{1}-$ $\hat{e}_{2}^{T} Z_{1} \hat{e}_{2}+\hat{e}_{2}^{T} Z_{2} \hat{e}_{2}-\hat{e}_{3}^{T} Z_{2} \hat{e}_{3}+\operatorname{Sym}\left\{-\hat{e}_{1}^{T}\left(I_{N} \otimes F\right) \hat{e}_{13}+\hat{e}_{1}^{T}\left(I_{N} \otimes\right.\right.$ 
$F A) \hat{e}_{1}+\hat{e}_{1}^{T}\left(I_{N} \otimes F B\right) \hat{e}_{4}+\hat{e}_{1}^{T}\left(I_{N} \otimes F C\right) \hat{e}_{5}-\hat{e}_{1}^{T}\left(G_{i} \otimes J_{i}\right) \hat{e}_{16}-$ $\left.\hat{e}_{1}^{T}\left(G_{i} \otimes J_{i}\right) \hat{e}_{17}\right\}+\operatorname{Sym}\left\{-m \hat{e}_{13}^{T}\left(I_{N} \otimes F\right) \hat{e}_{13}+m \hat{e}_{13}^{T}\left(I_{N} \otimes\right.\right.$ $F A) \hat{e}_{1}+m \hat{e}_{13}^{T}\left(I_{N} \otimes F B\right) \hat{e}_{4}+m \hat{e}_{13}^{T}\left(I_{N} \otimes F C\right) \hat{e}_{5}+m \hat{e}_{13}^{T}\left(I_{N} \otimes\right.$ $\left.F B_{w}\right) \hat{e}_{18}-m \hat{e}_{13}^{T}\left(G_{i} \otimes J_{i}\right) \hat{e}_{16}-m \hat{e}_{13}^{T}\left(G_{i} \otimes J_{i}\right) \hat{e}_{17}+\hat{e}_{1}^{T}\left(I_{N} \otimes\right.$ $\left.\left.F B_{w}\right) \hat{e}_{18}\right\}-\hat{e}_{1}^{T}\left(I_{N} \otimes D\right)^{T} \mathcal{P}\left(I_{N} \otimes D\right) \hat{e}_{1}-\operatorname{Sym}\left\{\hat{e}_{1}^{T}\left(I_{N} \otimes\right.\right.$ $\left.D)^{T} \mathcal{T} \hat{e}_{18}\right\}-\hat{e}_{18}^{T}\left(\mathcal{R}-\rho I_{N n}\right) \hat{e}_{18}+\left(\hat{e}_{16}+\hat{e}_{17}\right)^{T}\left(G_{i}^{T} \Theta G_{i} \otimes\right.$ $\Omega)\left(\hat{e}_{16}+\hat{e}_{17}\right)-\hat{e}_{17}^{T}\left(I_{N} \otimes \Omega\right) \hat{e}_{17}+\eta_{1}^{T} \Xi_{1} \eta_{1}+\eta_{2}^{T} \Xi_{2} \eta_{2}+\eta_{3}^{T} \Xi_{3} \eta_{3}$, $\tilde{\Xi}_{i i}=\hat{e}_{1}^{T} \sum_{j \in S_{\mathrm{k}}^{i}}^{\mathcal{M}}\left[\underline{\pi}_{i j}\left(P_{j}-P_{i}\right)+2 \varpi_{i j} V_{i j}\right] \hat{e}_{1}, \zeta_{0}=\left.\zeta_{\delta}\right|_{\delta=0}$, $\zeta_{1}=\left.\zeta_{\delta}\right|_{\delta=1}$,

$\zeta_{\delta}=U_{1}+\varphi \Lambda_{1}^{T}\left(\delta U_{2}+(1-\delta) U_{3}\right) \Lambda_{1}+\operatorname{Sym}\left\{\Lambda_{1}^{T} U_{2} \Lambda_{2}+\right.$ $\left.\Lambda_{1}^{T} U_{3} \Lambda_{3}\right\}+\frac{1}{\varphi}\left[\begin{array}{c}\Lambda_{2} \\ \Lambda_{3}\end{array}\right]^{T}\left[\begin{array}{cc}U_{2} & X_{1} \\ * & U_{3}\end{array}\right]\left[\begin{array}{l}\Lambda_{2} \\ \Lambda_{3}\end{array}\right]$,

$\Lambda_{1}=\left[\begin{array}{ll}\tilde{e}_{1}^{T} & 0\end{array}\right]^{T}, \Lambda_{2}=\left[\begin{array}{ll}0 & \tilde{e}_{3}^{T}\end{array}\right]^{T}, \Lambda_{3}=\left[\begin{array}{ll}0 & \tilde{e}_{4}^{T}\end{array}\right]^{T}$,

$\tilde{e}_{\alpha}=\left[0_{N n \times(\alpha-1) N n} I_{N n \times N n} 0_{N n \times(4-\alpha) N n}\right], \alpha=1,2,3,4$.

$\eta_{1}=\left[\hat{e}_{1}^{T}, \hat{e}_{4}^{T}\right], \eta_{2}=\left[\hat{e}_{2}^{T}, \hat{e}_{5}^{T}\right], \eta_{3}=\left[\hat{e}_{3}^{T}, \hat{e}_{6}^{T}\right], \delta=\frac{\varphi(t)}{\varphi}$,

$\hat{\phi}_{2}(\delta, 1-\delta)=\left[\begin{array}{cc}(2-\delta) \widehat{M}_{2} & (1-\delta) Y_{1}+\delta Y_{2} \\ * & (1+\delta) \widehat{M}_{2}\end{array}\right]$,

$\widehat{M}_{2}=\operatorname{diag}\left\{M_{2}, 3 M_{2}, 5 M_{2}, 7 M_{2}\right\}, \hat{\Theta}_{i}=\varpi_{i i}-\sum_{j \in S_{k}^{i}, j \neq i}^{\mathcal{M}} \varpi_{i j}$,

$\underline{\pi}_{i}=-\bar{\pi}_{i i}-\sum_{j \in S_{k}^{i}, j \neq i}^{\mathcal{M}} \underline{\pi}_{i j}, \underline{\pi}_{i j}=\tilde{\pi}_{i j}-\varpi_{i j}, \quad \bar{\pi}_{i j}=$ $\tilde{\pi}_{i j}+\varpi_{i j}, \Omega_{1}=\left[\hat{e}_{1}^{T}, \hat{e}_{2}^{T}, \varphi(t) \hat{e}_{7}^{T},(\varphi-\varphi(t)) \hat{e}_{8}^{T}\right]^{T}$, $\Omega_{2}=\left[\hat{e}_{13}^{T},(1-\dot{\varphi}(t)) \hat{e}_{14}^{T}, \hat{e}_{1}^{T}-(1-\dot{\varphi}(t)) \hat{e}_{2}^{T},(1-\right.$ $\left.\dot{\varphi}(t)) \hat{e}_{2}^{T}-\hat{e}_{3}^{T}\right]^{T}, \quad \Omega_{3}=\left[\hat{e}_{1}^{T}, \hat{e}_{7}^{T}\right]^{T}, \Omega_{4}=\left[\hat{e}_{1}^{T}, \hat{e}_{8}^{T}\right]^{T}$, $\Omega_{5}=\left[\delta \varphi \hat{e}_{13}^{T}, \hat{e}_{1}^{T}-(1-\dot{\varphi}(t)) \hat{e}_{2}^{T}-\dot{\varphi}(t) \hat{e}_{7}^{T}\right]^{T}$, $\Omega_{6}=\left[(1-\delta) \varphi \hat{e}_{13}^{T},(1-\dot{\varphi}(t)) \hat{e}_{2}^{T}-\hat{e}_{3}^{T}+\dot{\varphi}(t) \hat{e}_{8}^{T}\right]^{T}$,

$\Omega_{7}=\left[0, \hat{e}_{1}^{T}-\hat{e}_{2}^{T}, 0,-\hat{e}_{1}^{T}-\hat{e}_{2}^{T}+2 \hat{e}_{7}^{T}, 0, \hat{e}_{1}^{T}-\hat{e}_{2}^{T}+6 \hat{e}_{7}^{T}-6 \hat{e}_{9}^{T}\right]^{T}$, $\Omega_{8}=\left[\hat{e}_{7}^{T}, 0, \hat{e}_{7}^{T}-\hat{e}_{9}^{T}, 0, \hat{e}_{7}^{T}-3 \hat{e}_{9}^{T}+2 \hat{e}_{11}^{T}, 0\right]^{T}$,

$\Omega_{9}=\left[0, \hat{e}_{2}^{T}-\hat{e}_{3}^{T}, 0,-\hat{e}_{2}^{T}-\hat{e}_{3}^{T}+2 \hat{e}_{8}^{T}, 0, \hat{e}_{2}^{T}-\hat{e}_{3}^{T}+6 \hat{e}_{8}^{T}-6 \hat{e}_{10}^{T}\right]^{T}$, $\Omega_{10}=\left[\hat{e}_{8}^{T}, 0, \hat{e}_{8}^{T}-\hat{e}_{10}^{T}, 0, \hat{e}_{8}^{T}-3 \hat{e}_{10}^{T}+2 \hat{e}_{12}^{T}, 0\right]^{T}$, $\Omega_{11}=\left[\hat{e}_{1}^{T}, \hat{e}_{13}^{T}\right]^{T}, \Omega_{12}=\left[\hat{e}_{2}^{T}, \hat{e}_{14}^{T}\right]^{T}, \Omega_{13}=\left[\hat{e}_{3}^{T}, \hat{e}_{15}^{T}\right]^{T}$, $\tilde{w}_{1}=\left[\left(\hat{e}_{1}-\hat{e}_{2}\right)^{T},\left(\hat{e}_{1}+\hat{e}_{2}-2 \hat{e}_{7}\right)^{T},\left(\hat{e}_{1}-\hat{e}_{2}+6 \hat{e}_{7}-\right.\right.$ $\left.\left.6 \hat{e}_{9}\right)^{T},\left(\hat{e}_{1}+\hat{e}_{2}-12 \hat{e}_{7}+30 \hat{e}_{9}-20 \hat{e}_{11}\right)^{T}\right]^{T}, \tilde{w}_{2}=$ $\left[\left(\hat{e}_{2}-\hat{e}_{3}\right)^{T},\left(\hat{e}_{2}+\hat{e}_{3}-2 \hat{e}_{8}\right)^{T},\left(\hat{e}_{2}-\hat{e}_{3}+6 \hat{e}_{8}-\right.\right.$ $\left.\left.6 \hat{e}_{10}\right)^{T},\left(\hat{e}_{2}+\hat{e}_{3}-12 \hat{e}_{8}+30 \hat{e}_{10}-20 \hat{e}_{12}\right)^{T}\right]^{T}, Q_{31}=$ $Q_{3 p}, Q_{41}=Q_{4 p}, Q_{32}=Q_{3 p}-\varphi Q_{3 q}, Q_{42}=Q_{4 p}+\varphi Q_{4 q}$, $N_{1,1}=\left.N_{1}\right|_{\dot{\varphi}(t)=\mu_{1}}, N_{1,2}=\left.N_{1}\right|_{\dot{\varphi}(t)=\mu_{2}}, N_{2,1}=\left.N_{2}\right|_{\dot{\varphi}(t)=\mu_{1}}$, $N_{2,2}=\left.N_{2}\right|_{\dot{\varphi}(t)=\mu_{2}}, N_{1}=M_{1}+\dot{\varphi}(t) Q_{3 q}+\left[\begin{array}{cc}0 & Z_{1} \\ Z_{1} & 0\end{array}\right]$, $N_{2}=M_{1}+\dot{\varphi}(t) Q_{4 q}+\left[\begin{array}{cc}0 & Z_{2} \\ Z_{2} & 0\end{array}\right], \quad \hat{Q}_{3 q}=$ $\operatorname{diag}\left\{N_{1}, 3 N_{1}, 5 N_{1}\right\}, \quad \hat{Q}_{4 q}=\operatorname{diag}\left\{N_{2}, 3 N_{2}, 5 N_{2}\right\}$, $\Xi_{\alpha_{1}}=\left[\begin{array}{cc}-R_{\alpha_{1}} \bar{E}_{1} & R_{\alpha_{1}} \bar{E}_{2} \\ * & -R_{\alpha_{1}}\end{array}\right]\left(\alpha_{1}=1,2,3\right)$.

If $i \in S_{\mathrm{k}}^{i}$,

$$
\begin{gathered}
P_{j}-P_{i}-V_{i j} \leq 0(\forall j \in \mathcal{S}, j \neq i), \\
{\left[\begin{array}{cc}
\breve{\Phi}\left(\mu_{1}, 0\right) & \tilde{w}_{1}^{T} Y_{2} \\
* & -\widehat{M}_{2}
\end{array}\right]<0,} \\
{\left[\begin{array}{cc}
\breve{\Phi}\left(\mu_{1}, \varphi\right) & \tilde{w}_{2}^{T} Y_{1}^{T} \\
* & -\widehat{M}_{2}
\end{array}\right]<0,} \\
{\left[\begin{array}{cc}
\breve{\Phi}\left(\mu_{2}, 0\right) & \tilde{w}_{1}^{T} Y_{2} \\
* & -\widehat{M}_{2}
\end{array}\right]<0,}
\end{gathered}
$$

$$
\begin{array}{r}
{\left[\begin{array}{cc}
\breve{\Phi}\left(\mu_{2}, \varphi\right) & \tilde{w}_{2}^{T} Y_{1}^{T} \\
* & -\widehat{M}_{2}
\end{array}\right]<0,} \\
{\left[\begin{array}{cc}
U_{2} & X_{1} \\
* & U_{3}
\end{array}\right] \geq 0,\left[\begin{array}{cc}
\hat{Q}_{3 q} & X_{2} \\
* & \hat{Q}_{4 q}
\end{array}\right] \geq 0,}
\end{array}
$$

$\zeta_{0}>0, \zeta_{1}>0, Q_{k, l}>0, N_{\rho, l}>0(k=3,4, \rho, l=1,2)$.

where

$\breve{\Phi}\left(\mu_{1}, 0\right)=\left.\Xi(\dot{\varphi}(t), \varphi(t))\right|_{\dot{\varphi}(t)=\mu_{1}, \varphi(t)=0}+\hat{\Xi}_{t}+\breve{\Xi}_{i i}$,

$\breve{\Phi}\left(\mu_{1}, \varphi\right)=\left.\Xi(\dot{\varphi}(t), \varphi(t))\right|_{\dot{\varphi}(t)=\mu_{1}, \varphi(t)=\varphi}+\hat{\Xi}_{t}+\breve{\Xi}_{i i}$,

$\breve{\Phi}\left(\mu_{2}, 0\right)=\left.\Xi(\dot{\varphi}(t), \varphi(t))\right|_{\dot{\varphi}(t)=\mu_{2}, \varphi(t)=0}+\hat{\Xi}_{t}+\breve{\Xi}_{i i}$,

$\breve{\Phi}\left(\mu_{2}, \varphi\right)=\left.\Xi(\dot{\varphi}(t), \varphi(t))\right|_{\dot{\varphi}(t)=\mu_{2}, \varphi(t)=\varphi}+\hat{\Xi}_{t}+\breve{\Xi}_{i i}$,

In matrices $\breve{\Phi}\left(\mu_{1}, 0\right), \breve{\Phi}\left(\mu_{2}, 0\right), \breve{\Phi}\left(\mu_{1}, \varphi\right)$ and $\breve{\Phi}\left(\mu_{2}, \varphi\right)$, only the element $\breve{\Xi}_{i i}$ is different from $\Xi_{i i}$, and the other elements are the same as the elements in $\tilde{\Phi}\left(\mu_{1}, 0\right), \tilde{\Phi}\left(\mu_{2}, 0\right), \tilde{\Phi}\left(\mu_{1}, \varphi\right)$ and $\tilde{\Phi}\left(\mu_{2}, \varphi\right)$.

$\breve{\Xi}_{i i}=\hat{e}_{1}^{T}\left[\sum_{j \in S_{k}^{i}, j \neq i}^{\mathcal{M}}\left[\underline{\pi}_{i j}\left(P_{j}-P_{i}\right)+2 \varpi_{i j} V_{i j}\right]\right.$

$\left.+\underline{\pi}_{i}\left(P_{\varepsilon}-P_{i}\right)+2 \hat{\Theta}_{i} V_{i \varepsilon}\right] \hat{e}_{1},\left(\varepsilon \in S_{\mathrm{uk}}^{i}\right)$.

Moreover, the feedback gain matrices are given as follows

$$
K_{i}=F^{-1} J_{i}(i \in \mathcal{S}) .
$$

Proof From Theorem 1, the stochastic consensus of error system (9) with $w_{k}(t)=0$ is reached based on (13)-(27). Under zero initial condition and $w_{k}(t) \neq 0$, the dissipative capacity analysis of system (9) is given in this section.

Let $\varsigma(t)=\left[\xi^{T}(t), w^{T}(t)\right]^{T}$, from (29) to (62), one obtains

$$
\begin{aligned}
& \mathcal{L} V(t, i)-h^{T}(t) \mathcal{P} h(t)-2 h^{T}(t) \mathcal{T} w(t)-w^{T}(t)(\mathcal{R} \\
& \left.-\rho I_{N n}\right) w(t) \\
& \leq \varsigma^{T}(t) \hat{\wp}(t) \varsigma(t)-\delta \tilde{w}_{2}^{T} Y_{1}^{T} \widehat{M}_{2}^{-1} Y_{1} \tilde{w}_{2}+(1 \\
& -\delta) \tilde{w}_{1}^{T} Y_{2} \widehat{M}_{2}^{-1} Y_{2}^{T} \tilde{w}_{1},
\end{aligned}
$$

where

$$
\hat{\wp}(t)=\hat{\Xi}(\dot{\varphi}(t), \varphi(t))+\hat{\Xi}_{t}+\hat{e}_{1}^{T} \sum_{j=1}^{\mathcal{M}} \pi_{i j} P_{j} \hat{e}_{1} .
$$

From (82)-(96), Schur complement lemma, and the proof of Theorem 1, it implies that

$$
\begin{aligned}
& \mathcal{L} V(t, i) \\
& \leq h^{T}(t) \mathcal{P} h(t)+2 h^{T}(t) \mathcal{T} w(t)+w^{T}(t)\left(\mathcal{R}-\rho I_{N n}\right) w(t) .
\end{aligned}
$$

For (100), integrating $[0, \sigma]$, one obtains

$$
\begin{aligned}
& \int_{0}^{\sigma}\{\mathcal{L} V(\alpha, i)\} d \alpha \\
& \leq \int_{0}^{\sigma} h^{T}(\alpha) \mathcal{P} h(\alpha) d \alpha+\int_{0}^{\sigma} 2 h^{T}(\alpha) \mathcal{T} w(\alpha) d \alpha \\
& +\int_{0}^{\sigma} w^{T}(\alpha)\left(\mathcal{R}-\rho I_{N n}\right) w(\alpha) d \alpha .
\end{aligned}
$$

According to Dynkin's formula, one has

$$
\mathcal{E}\{V(\sigma, i)\} \leq \mathcal{E}\langle h, \mathcal{P} h\rangle_{\sigma}+2 \mathcal{E}\langle h, \mathcal{T} w\rangle_{\sigma}
$$




$$
+\mathcal{E}\left\langle w,\left(\mathcal{R}-\rho I_{N n}\right) w\right\rangle_{\sigma}
$$

For any $\sigma>0$, we have

$$
\mathcal{E}\langle h, \mathcal{P} h\rangle_{\sigma}+2 \mathcal{E}\langle h, \mathcal{T} w\rangle_{\sigma}+\mathcal{E}\langle w, \mathcal{R} w\rangle_{\sigma} \geq \rho \mathcal{E}\langle w, w\rangle_{\sigma}
$$

Thus, the strictly $(\mathcal{P}, \mathcal{T}, \mathcal{R})-\rho$-dissipative capacity of the leader-following error system (9) is achieved. From Definitions 1-2, the leader-following dissipativity consensus issue of NMASs (2) and (3) is solved by consensus protocol (5). According to Theorem 1, we obtain the following feedback gain matrices

$$
K_{i}=F^{-1} J_{i}(i \in \mathcal{S})
$$

Remark 6: Based on DPTLKF and event-triggered strategy, the dissipativity-based consensus issue of delayed NMASs with generally uncertain Markovian switching topologies is investigated for the first time in this article. In addition, more information about time-varying delay and its derivative is contained in the dissipativity-based consensus conditions by utilizing DPTLKF and HOPRII.

Remark 7: In view of [63] and [51], $H_{\infty}$ criteria could be obtained by setting $\mathcal{P}=-I, \mathcal{T}=0, \mathcal{R}-\rho=\gamma^{2} I$, and the passivity problem could be obtained by setting $\mathcal{P}=0, \mathcal{T}=I$, $\mathcal{R}-\rho=\gamma I$.

Remark 8: Because of the uncertainty disturbance and the weak communication of current and wave on the sea, the Markovian switching topologies and event-triggered transmission strategy in this article could be used in remotely operated underwater vehicles (ROVs). As a kinds of NMSs, the tracking control of ROVs was investigated [64] based on the motion model $\bar{M}_{k} \ddot{\eta}_{k}+\bar{C}_{k}\left(\dot{\eta}_{k}\right) \dot{\eta}_{k}+\bar{D}_{k}\left(\dot{\eta}_{k}\right) \dot{\eta}_{k}+\bar{g}_{k}\left(\eta_{k}\right)=$ $\tau_{k}(k=1,2, \cdots, N) . k=1$ is selected as the leader agent, whose aim is to track the target point. Meanwhile, other ROVs are selected as the following agents, which keeps a desired relative state from the leader agent. Based on the method in this article and the following ROVs, the distributed controller based on Markovian switching topologies could be designed as $\tau_{k}=-K\left(r_{t}\right)\left\{\sum_{l \in \mathbb{N}_{k}} a_{k l}\left(r_{t}\right)\left[\eta_{k}\left(t_{p}^{k}\right)-\eta_{l}\left(t_{\hat{p}}^{l}\right)\right]+\gamma_{k}\left[\eta_{k}\left(t_{p}^{k}\right)-\right.\right.$ $\left.\left.\eta_{1}(q h)\right]\right\}(k=2, \cdots, N)$. To save the network communication resources and achieve tracking control of ROVs efficiently, the distributed event-triggered strategy for each following ROV could be designed as $\left[y_{k}\left(t_{p}^{k}+\beta h\right)\right]^{T} \Omega y_{k}\left(t_{p}^{k}+\beta h\right) \leq$ $\theta_{k}\left[w_{k}\left(t_{p}^{k}+\beta h\right)\right]^{T} \Omega w_{k}\left(t_{p}^{k}+\beta h\right)$. When we establish appropriate Lyapunov-Krasovskii functional, the corresponding position tracking control conditions of the ROVs could be obtained based on Markovian switching topologies and eventtriggered transmission strategy.

From Theorem 2, the dissipativity-based consensus tracking design algorithm is given in algorithm 1 .

\section{Algorithm 1 Dissipativity-based consensus tracking design}

Step 1 Calculate the delay parameters $\mu_{1}, \mu_{2}, \varphi$;

Step 2 Set the scalar $m=m^{*}$, the event-triggered parameters

$\theta_{\varrho}=\theta_{\varrho}^{*}(\varrho=1,2,3,4)$, the transition rates $\varpi_{\kappa i}=\varpi_{\kappa i}^{*}$

$(\kappa=1,2,3), \rho=\rho^{*}$, and the matrices $\mathcal{P}=\mathcal{P}^{*}, \mathcal{T}=\mathcal{T}^{*}$,

$\mathcal{R}=\mathcal{R}^{*}$;

Step 3 If LMIs (82)-(96) in Theorem 2 are feasible,

obtain matrices $F, J_{i}(i \in \mathcal{S})$. Otherwise, go to Step 2;

Step 4 Compute the designed controller gains

$K_{i}=F^{-1} J_{i}(i \in \mathcal{S})$;

Step 5 Select the exogenous disturbance $w_{k}(t)$

$(k=1,2, \cdots, N)$, and plot the error state curves

and event-triggered moments of the nonlinear error

dynamics system (9).

\section{Simulation}

In order to verify the proposed results, two simulation examples based on the generally uncertain Markovian switching topologies and event-triggered strategy are presented in this section.

Example 1 Consider the consensus tracking control of the following time-varying NMASs

$$
\left\{\begin{array}{c}
\dot{x}_{k}(t)=A x_{k}(t)+B f\left(x_{k}(t)\right)+C f\left(x_{k}(t-\varphi(t))\right. \\
+u_{k}(t)+B_{w} w_{k}(t) \\
\psi_{k}(t)=D x_{k}(t), k=1,2, \cdots, 7 .
\end{array}\right.
$$

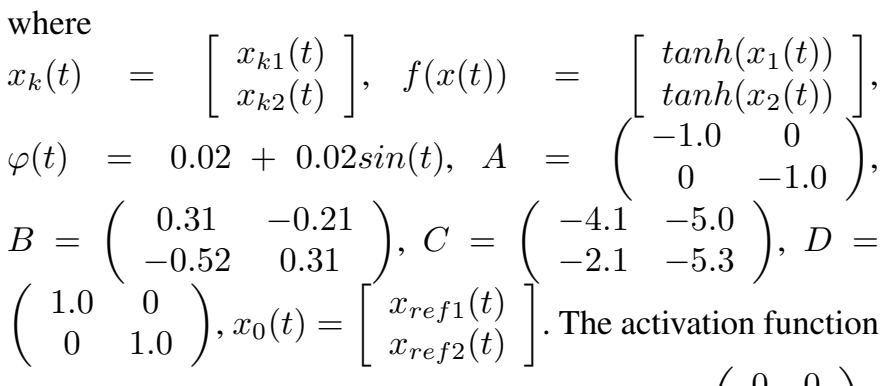
satisfies Assumption 3, and we obtain $E_{1}=\left(\begin{array}{ll}0 & 0 \\ 0 & 0\end{array}\right)$, $E_{2}=\left(\begin{array}{cc}0.5 & 0 \\ 0 & 0.5\end{array}\right)$. From time-varying delay $\varphi(t)$, we obtain $\varphi=0.04, \mu_{1}=-0.02, \mu_{2}=0.02$.

With $w_{k}(t)=0$, this example focuses on the consensus tracking control problem. The communication network between the agents is expressed as a set of Markovian switching topologies in Fig 2, which is dominated by a generally uncertain Markovian switching process with three modes. The leader agent is denoted as 0 . The follower agents are denoted as $1,2, \cdots, 7$.

According to Fig. 2, the Laplacian matrices $\mathbb{L}_{i}$ and the leader adjacency matrices $\Gamma_{i}(i=1,2,3)$ are obtained as follows 
$\mathbb{L}_{1}=\left(\begin{array}{ccccccc}0 & 0 & 0 & 0 & 0 & 0 & 0 \\ 0 & 0 & 0 & 0 & 0 & 0 & 0 \\ -1 & 0 & 1 & 0 & 0 & 0 & 0 \\ -1 & 0 & 0 & 1 & 0 & 0 & 0 \\ 0 & 0 & -1 & 0 & 1 & 0 & 0 \\ 0 & -1 & 0 & 0 & 0 & 1 & 0 \\ 0 & -1 & 0 & 0 & 0 & 0 & 1\end{array}\right)$,

$\Gamma_{1}=\operatorname{diag}\{1,1,0,0,0,0,0\}, \Gamma_{2}=\operatorname{diag}\{1,0,1,0,0,0,0\}$, $\Gamma_{3}=\operatorname{diag}\{1,0,0,1,0,0,0\}$.

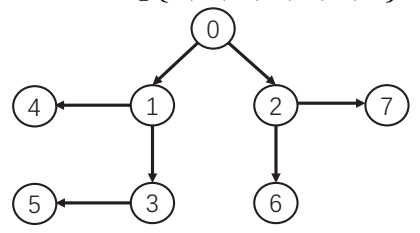

2.1

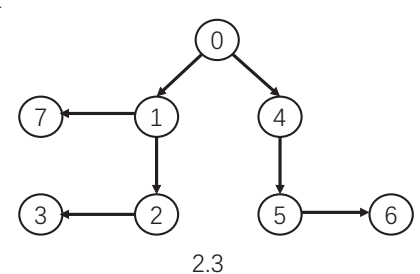

Fig. 2 The generally uncertain Markovian switching topologies.

The transition matrix is given as follows

$\Pi=\left(\begin{array}{ccc}? & ? & 2.51+\triangle_{13} \\ ? & -4.67+\triangle_{22} & ? \\ ? & ? & -3.13+\triangle_{33}\end{array}\right)$, where $\varpi_{1 i}=$ $0.191, \varpi_{2 i}=0.219, \varpi_{3 i}=0.138$

The event-triggered parameters are given as $\theta_{1}=0.08$, $\theta_{2}=0.04, \theta_{3}=0.11, \theta_{4}=0.14, \theta_{5}=0.09, \theta_{6}=$ $0.07, \theta_{7}=0.1$. We obtain the following consensus feedback matrices by adopting Matlab LMI Toolbox. $K_{1}=$ $\left(\begin{array}{cc}0.0637 & -0.0221 \\ -0.0012 & 0.0666\end{array}\right), K_{2}=\left(\begin{array}{cc}0.0622 & -0.0177 \\ 0.0061 & 0.0719\end{array}\right)$, $K_{3}=\left(\begin{array}{cc}0.0708 & -0.0236 \\ 0.0003 & 0.0809\end{array}\right)$.

The initial conditions of the followers and the leader are given as $x_{1}(t)=\left[\begin{array}{ll}0.3 & 0.8\end{array}\right]^{T}, x_{2}(t)=\left[\begin{array}{ll}0.6 & 0.3\end{array}\right]^{T}$, $x_{3}(t)=\left[\begin{array}{ll}0.3 & 0.3\end{array}\right]^{T}, \quad x_{4}(t)=\left[\begin{array}{ll}0.5 & 0.6\end{array}\right]^{T}$,
$x_{5}(t)=\left[\begin{array}{ll}0.8 & 0.2\end{array}\right]^{T}, \quad x_{6}(t)=\left[\begin{array}{ll}0.4 & 0.3\end{array}\right]^{T}$,
$x_{7}(t)=\left[\begin{array}{ll}0.7 & 0.6\end{array}\right]^{T}, x_{0}(t)=\left[\begin{array}{ll}0.2 & 0.1\end{array}\right]^{T}$.

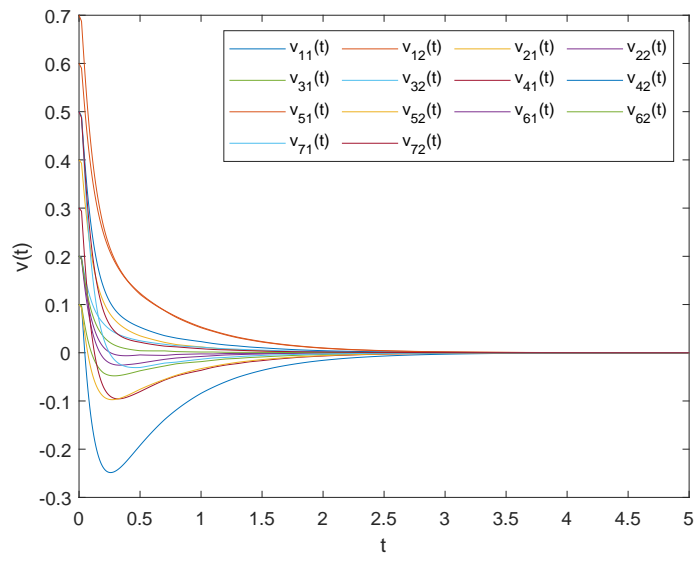

Fig. 3 The error states among the leader and the followers in Example 1.

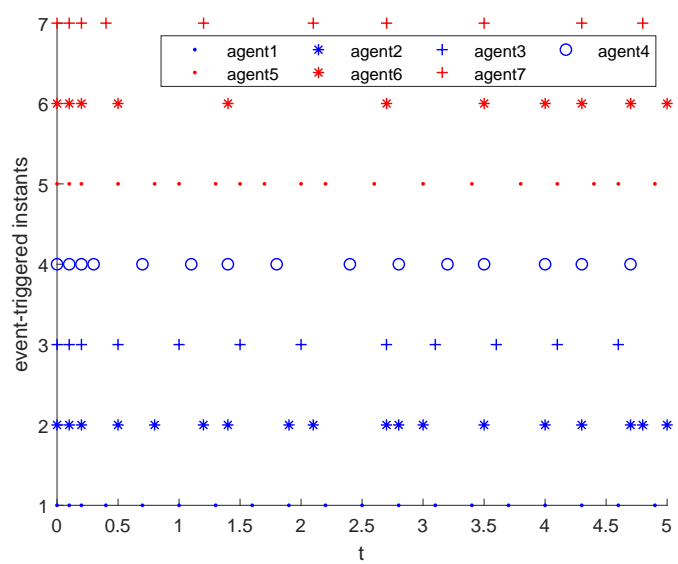

Fig. 4 The event-triggered moments for the followers in Example 1.

The error states are shown in Fig. 3, and the event-triggered instants are given in Fig. 4. From Figs. 2-4, the consensus tracking control could be achieved in leader NMAS (3) and following NMASs (105) with $w_{k}(t)=0$.

Example 2 With $w_{k}(t) \neq 0$, the dissipative capacity control issue of the leader-following NMASs (3) and (105) is proposed in this example. $B_{w}=\left(\begin{array}{ll}1 & 0 \\ 0 & 1\end{array}\right), w_{k}(t)=$ $\left(\begin{array}{l}0.01 \sin (t) \\ 0.01 \sin (t)\end{array}\right), \mathcal{P}=-5 * I_{14}, \mathcal{T}=-0.8 * I_{14}, \mathcal{R}=5 * I_{14}$. The initial conditions of the followers and the leader are given as $x_{1}(t)=\left[\begin{array}{ll}1.3 & 0.8\end{array}\right]^{T}, x_{2}(t)=\left[\begin{array}{ll}1.6 & 0.3\end{array}\right]^{T}$, $x_{3}(t)=\left[\begin{array}{ll}1.3 & 0.3\end{array}\right]^{T}, x_{4}(t)=\left[\begin{array}{ll}0.1 & 0.6\end{array}\right]^{T}$, $x_{5}(t)=\left[\begin{array}{ll}1.8 & 0.1\end{array}\right]^{T}, x_{6}(t)=\left[\begin{array}{ll}1.4 & 0.3\end{array}\right]^{T}, x_{7}(t)=$ $\left[\begin{array}{ll}0.1 & 0.6\end{array}\right]^{T}, x_{0}(t)=\left[\begin{array}{ll}0.3 & 0.2\end{array}\right]^{T}$. The generally Markovian switching topologies, event-triggered parameters and system parameters $A, B, C, D$ are given in example 1 .

By adopting Matlab LMI Toolbox, the consensus feedback matrices are obtained as follows $K_{1}=\left(\begin{array}{cc}0.1395 & 0.0019 \\ -0.0027 & 0.1401\end{array}\right), K_{2}=$ $\left(\begin{array}{ll}0.1298 & 0.0135 \\ 0.0229 & 0.1377\end{array}\right), \quad K_{3}=\left(\begin{array}{cc}0.1548 & 0.0076 \\ -0.0023 & 0.1716\end{array}\right)$. 


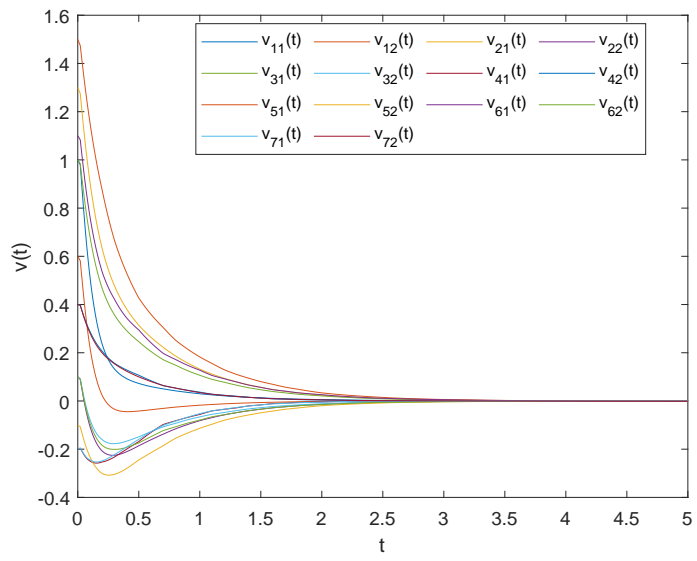

Fig. 5 The error states among the leader and the followers in Example 2.

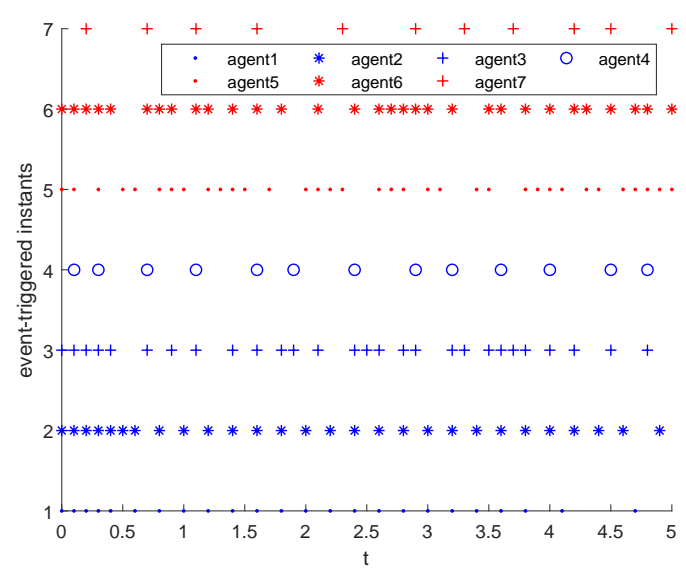

Fig. 6 The event-triggered moments for the followers in Example 2.

When $w_{k}(t) \neq 0$, the error states and event-triggered instants are given in Figs. 5-6. From Figs. 5-6 and Definitions 1-2, the dissipative consensus control is achieved by ETS (4) and consensus protocol (5).

\section{CONCLUSION}

In this article, the DBCTC problems of LFNMASs with time-varying delays have been investigated. Different from previous topologies, the Markovian switching topologies with uncertain and partially unknown transition rates are considered, which is more general. The ETS protocol has been established to reduce unnecessary communication when obtaining more information from the agent network. Based on DPTLKF and HOPRII, some consensus tracking control criteria have been proposed to guarantee leader-following consensus. Moreover, the consensus gain matrices have been obtained by solving the LMIs. Finally, two simulation examples have been presented to illustrate the validity of the theoretical results. In the future, we would like to focus on the distributed faulttolerant containment control of the delayed NMASs under adaptive event-triggered transmission strategy, as well as the consensus control of generally uncertain Markovian NMASs against denial-of-service attacks and actuator faults.

\section{REFERENCES}

[1] A. Bemporad and C. Rocchi, "Decentralized hybrid model predictive control of a formation of unmanned aerial vehicles," IFAC Proceedings Volumes, vol. 44, no. 1, pp. 11900-11906, 2011.

[2] C. P. Chen, G.-X. Wen, Y.-J. Liu, and F.-Y. Wang, "Adaptive consensus control for a class of nonlinear multiagent time-delay systems using neural networks," IEEE Transactions on Neural Networks and Learning Systems, vol. 25, no. 6, pp. 1217-1226, 2014.

[3] J. Dai and G. Guo, "Event-triggered leader-following consensus for multi-agent systems with semi-Markov switching topologies," Information Sciences, vol. 459, pp. 290-301, 2018.

[4] W. Zou, P. Shi, Z. Xiang, and Y. Shi, "Consensus tracking control of switched stochastic nonlinear multiagent systems via event-triggered strategy," IEEE Transactions on Neural Networks and Learning Systems, vol. 31, no. 3, pp. 1036-1045, 2020.

[5] Z. Cao, C. Li, X. Wang, and T. Huang, "Finite-time consensus of linear multi-agent system via distributed event-triggered strategy," Journal of the Franklin Institute, vol. 355, no. 3, pp. 1338-1350, 2018.

[6] H. Su, X. Wang, and Z. Zeng, "Consensus of second-order hybrid multiagent systems by event-triggered strategy," IEEE Transactions on Cybernetics, vol. 50, no. 11, pp. 4648-4657, 2020.

[7] G. Ren, Y. Yu, C. Xu, and X. Hai, "Consensus of fractional multi-agent systems by distributed event-triggered strategy," Nonlinear Dynamics, vol. 95, no. 1, pp. 541-555, 2019.

[8] W. He, B. Xu, Q.-L. Han, and F. Qian, "Adaptive consensus control of linear multiagent systems with dynamic event-triggered strategies," IEEE Transactions on Cybernetics, vol. 50, no. 7, pp. 2996-3008, 2020.

[9] C. Du, X. Liu, W. Ren, P. Lu, and H. Liu, "Finite-time consensus for linear multiagent systems via event-triggered strategy without continuous communication," IEEE Transactions on Control of Network Systems, vol. 7, no. 1, pp. 19-29, 2020.

[10] L. Ding, Q.-L. Han, X. Ge, and X.-M. Zhang, "An overview of recent advances in event-triggered consensus of multiagent systems," IEEE Transactions on Cybernetics, vol. 48, no. 4, pp. 1110-1123, 2018.

[11] H. Liang, L. Zhang, Y. Sun, and T. Huang, "Containment control of semi-markovian multiagent systems with switching topologies," IEEE Transactions on Systems, Man, and Cybernetics: Systems, vol. 51, no. 6, pp. 3889-3899, 2021.

[12] T. T. Nguyen, N. D. Nguyen, and S. Nahavandi, "Deep reinforcement learning for multiagent systems: A review of challenges, solutions, and applications," IEEE Transactions on Cybernetics, vol. 50, no. 9, pp. 3826-3839, 2020.

[13] Z. Zuo, Q.-L. Han, B. Ning, X. Ge, and X.-M. Zhang, "An overview of recent advances in fixed-time cooperative control of multiagent systems," IEEE Transactions on Industrial Informatics, vol. 14, no. 6, pp. 23222334, 2018.

[14] H. Zhang, Y. Zhou, Y. Liu, and J. Sun, "Cooperative bipartite containment control for multiagent systems based on adaptive distributed observer," IEEE Transactions on Cybernetics, doi:10.1109/TCYB.2020.3031933.

[15] H. Zhang, Y. Cai, Y. Wang, and H. Su, "Adaptive bipartite event-triggered output consensus of heterogeneous linear multiagent systems under fixed and switching topologies," IEEE Transactions on Neural Networks and Learning Systems, vol. 31, no. 11, pp. 4816-4830, 2020.

[16] H. Zhang, J. Duan, Y. Wang, and Z. Gao, "Bipartite fixed-time output consensus of heterogeneous linear multiagent systems," IEEE Transactions on Cybernetics, vol. 51, no. 2, pp. 548-557, 2021.

[17] S. Preitl, R.-E. Precup, J. Fodor, and B. Bede, "Iterative feedback tuning in fuzzy control systems. theory and applications," Acta Polytechnica Hungarica, vol. 3, no. 3, pp. 81-96, 2006.

[18] P. De Wilde and G. Briscoe, "Stability of evolving multiagent systems," IEEE Transactions on Systems, Man, and Cybernetics, Part B (Cybernetics), vol. 41, no. 4, pp. 1149-1157, 2011.

[19] R.-E. Precup, T.-A. Teban, A. Albu, A.-B. Borlea, I. A. Zamfirache, and E. M. Petriu, "Evolving fuzzy models for prosthetic hand myoelectricbased control," IEEE Transactions on Instrumentation and Measurement, vol. 69, no. 7, pp. 4625-4636, 2020.

[20] A. Turnip and J. H. Panggabean, "Hybrid controller design based magneto-rheological damper lookup table for quarter car suspension," International Journal of Artificial Intelligence, vol. 18, no. 1, pp. 193206, 2020.

[21] D. Yu and C. P. Chen, "Automatic leader-follower persistent formation generation with minimum agent-movement in various switching topologies," IEEE Transactions on Cybernetics, vol. 50, no. 4, pp. 1569-1581, 2020. 
[22] R.-C. Roman, R.-E. Precup, and E. M. Petriu, "Hybrid data-driven fuzzy active disturbance rejection control for tower crane systems," European Journal of Control, vol. 58, pp. 373-387, 2021.

[23] G. Wen, Y. Zhao, Z. Duan, W. Yu, and G. Chen, "Containment of higherorder multi-leader multi-agent systems: A dynamic output approach," IEEE Transactions on Automatic Control, vol. 61, no. 4, pp. 1135-1140, 2016.

[24] X. Tan, J. Cao, and X. Li, "Consensus of leader-following multiagent systems: A distributed event-triggered impulsive control strategy," IEEE Transactions on Cybernetics, vol. 49, no. 3, pp. 792-801, 2018.

[25] H. A. Hashim, S. El-Ferik, and F. L. Lewis, "Neuro-adaptive cooperative tracking control with prescribed performance of unknown higher-order nonlinear multi-agent systems," International Journal of Control, vol. 92, no. 2, pp. 445-460, 2019.

[26] J. Hu and J. Cao, "Hierarchical cooperative control for multiagent systems with switching directed topologies," IEEE Transactions on Neural Networks and Learning Systems, vol. 26, no. 10, pp. 2453-2463, 2015.

[27] X. Ge, Q.-L. Han, D. Ding, X.-M. Zhang, and B. Ning, "A survey on recent advances in distributed sampled-data cooperative control of multiagent systems," Neurocomputing, vol. 275, pp. 1684-1701, 2018.

[28] H. Yang, Q.-L. Han, X. Ge, L. Ding, Y. Xu, B. Jiang, and D. Zhou, "Faulttolerant cooperative control of multiagent systems: A survey of trends and methodologies,' IEEE Transactions on Industrial Informatics, vol. 16, no. 1, pp. 4-17, 2020.

[29] Q. Song, J. Cao, and W. Yu, "Second-order leader-following consensus of nonlinear multi-agent systems via pinning control," Systems \& Control Letters, vol. 59, no. 9, pp. 553-562, 2010.

[30] L. Cheng, Z.-G. Hou, M. Tan, Y. Lin, and W. Zhang, "Neural-networkbased adaptive leader-following control for multiagent systems with uncertainties," IEEE Transactions on Neural Networks, vol. 21, no. 8, pp. 1351-1358, 2010 .

[31] H. Du, G. Wen, G. Chen, J. Cao, and F. E. Alsaadi, "A distributed finite-time consensus algorithm for higher-order leaderless and leaderfollowing multiagent systems," IEEE Transactions on Systems, Man, and Cybernetics: Systems, vol. 47, no. 7, pp. 1625-1634, 2017.

[32] W. Zhu and Z.-P. Jiang, "Event-based leader-following consensus of multi-agent systems with input time delay," IEEE Transactions on Automatic Control, vol. 60, no. 5, pp. 1362-1367, 2015.

[33] H. Li, X. Liao, T. Huang, and W. Zhu, "Event-triggering sampling based leader-following consensus in second-order multi-agent systems," IEEE Transactions on Automatic Control, vol. 60, no. 7, pp. 1998-2003, 2015.

[34] X.-Z. Jin, Z. Zhao, and Y.-G. He, "Insensitive leader-following consensus for a class of uncertain multi-agent systems against actuator faults," Neurocomputing, vol. 272, pp. 189-196, 2018.

[35] Z. Zuo, Q.-L. Han, and B. Ning, "An explicit estimate for the upper bound of the settling time in fixed-time leader-following consensus of high-order multivariable multiagent systems," IEEE Transactions on Industrial Electronics, vol. 66, no. 8, pp. 6250-6259, 2019.

[36] S. Wang, Y. Wang, Y. Jiang, and Y. Li, "Event-triggered based distributed $H_{\infty}$ consensus filtering for discrete-time delayed systems over lossy sensor network," Transactions of the Institute of Measurement and Control, vol. 40, no. 9, pp. 2740-2747, 2018.

[37] D. V. Dimarogonas, E. Frazzoli, and K. H. Johansson, "Distributed event-triggered control for multi-agent systems," IEEE Transactions on Automatic Control, vol. 57, no. 5, pp. 1291-1297, 2012.

[38] W. Hu, L. Liu, and G. Feng, "Consensus of linear multi-agent systems by distributed event-triggered strategy," IEEE Transactions on Cybernetics, vol. 46, no. 1, pp. 148-157, 2016.

[39] Z.-G. Wu, Y. Xu, R. Lu, Y. Wu, and T. Huang, "Event-triggered control for consensus of multiagent systems with fixed/switching topologies," IEEE Transactions on Systems, Man, and Cybernetics: Systems, vol. 48, no. 10, pp. 1736-1746, 2018.

[40] B. Cheng, X. Wang, and Z. Li, "Event-triggered consensus of homogeneous and heterogeneous multiagent systems with jointly connected switching topologies," IEEE Transactions on Cybernetics, vol. 49, no. 12, pp. 4421-4430, 2019.

[41] M. Chen, H. Yan, H. Zhang, S. Chen, and Z. Li, "Event-triggered consensus of multiagent systems with time-varying communication delay," IEEE Transactions on Systems, Man, and Cybernetics: Systems, doi: 10.1109/TSMC.2021.3051396.

[42] W. Yu, G. Chen, M. Cao, and J. Kurths, "Second-order consensus for multiagent systems with directed topologies and nonlinear dynamics," IEEE Transactions on Systems, Man, and Cybernetics, Part B (Cybernetics), vol. 40, no. 3, pp. 881-891, 2010.

[43] Y. Zhao, T. Wang, and W. Bi, "Consensus protocol for multiagent systems with undirected topologies and binary-valued communications,"
IEEE Transactions on Automatic Control, vol. 64, no. 1, pp. 206-221, 2019.

[44] J. Dai and G. Guo, "Exponential consensus of non-linear multi-agent systems with semi-Markov switching topologies," IET Control Theory \& Applications, vol. 11, no. 18, pp. 3363-3371, 2017.

[45] L. Ding and G. Guo, "Sampled-data leader-following consensus for nonlinear multi-agent systems with Markovian switching topologies and communication delay," Journal of the Franklin Institute, vol. 352, no. 1, pp. 369-383, 2015.

[46] K. You, Z. Li, and L. Xie, "Consensus condition for linear multi-agent systems over randomly switching topologies," Automatica, vol. 49, no. 10, pp. 3125-3132, 2013

[47] Y. Zhang and Y.-P. Tian, "Consentability and protocol design of multiagent systems with stochastic switching topology," Automatica, vol. 45, no. 5, pp. 1195-1201, 2009.

[48] J. Wang, H. Zhang, Z. Wang, and Z. Liu, "Sampled-data synchronization of Markovian coupled neural networks with mode delays based on modedependent LKF," IEEE Transactions on Neural Networks and Learning Systems, vol. 28, no. 11, pp. 2626-2637, 2017.

[49] J. Wang, H. Zhang, Z. Wang, and Q. Shan, "Local synchronization criteria of markovian nonlinearly coupled neural networks with uncertain and partially unknown transition rates," IEEE Transactions on Systems, Man, and Cybernetics: Systems, vol. 47, no. 8, pp. 1953-1964, 2017.

[50] Z.-G. Wu, J. Lam, H. Su, and J. Chu, "Stability and dissipativity analysis of static neural networks with time delay," IEEE Transactions on Neural Networks and Learning Systems, vol. 23, no. 2, pp. 199-210, 2012.

[51] X. Jiang, G. Xia, Z. Feng, Z. Jiang, and Z.-G. Wu, "Dissipativitybased consensus tracking of singular multiagent systems with switching topologies and communication delays," IEEE Transactions on Cybernetics, doi: $10.1109 /$ TCYB.2020.3030037.

[52] Y. Xu, R. Lu, H. Peng, K. Xie, and A. Xue, "Asynchronous dissipative state estimation for stochastic complex networks with quantized jumping coupling and uncertain measurements," IEEE Transactions on Neural Networks and Learning Systems, vol. 28, no. 2, pp. 268-277, 2017.

[53] P. Shi, X. Su, and F. Li, "Dissipativity-based filtering for fuzzy switched systems with stochastic perturbation," IEEE Transactions on Automatic Control, vol. 61, no. 6, pp. 1694-1699, 2016.

[54] Y. Wu, Z.-H. Guan, and T. Li, "Dissipative consensus problems for multi-agent networks via sliding mode control," Journal of the Franklin Institute, vol. 354, no. 14, pp. 6282-6291, 2017.

[55] P. Shi and J. Yu, "Dissipativity-based consensus for fuzzy multiagent systems under switching directed topologies," IEEE Transactions on Fuzzy Systems, vol. 29, no. 5, pp. 1143-1151, 2021

[56] L. Ding and W. X. Zheng, "Network-based practical consensus of heterogeneous nonlinear multiagent systems," IEEE Transactions on Cybernetics, vol. 47, no. 8, pp. 1841-1851, 2017.

[57] Z. Ye, H. Ji, and H. Zhang, "Passivity analysis of markovian switching complex dynamic networks with multiple time-varying delays and stochastic perturbations," Chaos, Solitons \& Fractals, vol. 83, pp. 147157,2016

[58] K. Liu and E. Fridman, "Wirtinger's inequality and Lyapunov-based sampled-data stabilization," Automatica, vol. 48, no. 1, pp. 102-108, 2012.

[59] R. Datta, R. Dey, B. Bhattacharya, R. Saravanakumar, and O. M. Kwon, "Stability and stabilization of T-S fuzzy systems with variable delays via new Bessel-Legendre polynomial based relaxed integral inequality,' Information Sciences, vol. 522, pp. 99-123, 2020.

[60] P. Park, W. I. Lee, and S. Y. Lee, "Auxiliary function-based integral inequalities for quadratic functions and their applications to time-delay systems," Journal of the Franklin Institute, vol. 352, no. 4, pp. 13781396.

[61] P. Park, J. W. Ko, and C. Jeong, "Reciprocally convex approach to stability of systems with time-varying delays," Automatica, vol. 47, no. 1, pp. 235-238.

[62] J. Wang, Z. Wang, X. Chen, and J. Qiu, "Synchronization criteria of delayed inertial neural networks with generally markovian jumping," Neural Networks, vol. 139, pp. 64-76, 2021.

[63] Z. Feng, J. Lam, and H. Gao, " $\alpha$-dissipativity analysis of singular timedelay systems," Automatica, vol. 47, no. 11, pp. 2548-2552, 2011.

[64] J. Yan, J. Gao, X. Yang, X. Luo, and X. Guan, "Position tracking control of remotely operated underwater vehicles with communication delay," IEEE Transactions on Control Systems Technology, vol. 28, no. 6, pp. 2506-2514, 2020 